Check for updates

Cite this: Mater. Chem. Front. 2020, 4, 2610

Received 26th March 2020

Accepted 24th June 2020

DOI: 10.1039/d0qm00180e

rsc.li/frontiers-materials

\section{Effect of pore structure and doping species on charge storage mechanisms in porous carbon-based supercapacitors}

\author{
Lijing Xie, ${ }^{a}$ Fangyuan Su, ${ }^{a}$ Longfei Xie, ${ }^{b}$ Xiaoqian Guo, ${ }^{a}$ Zhenbing Wang, ${ }^{a}$ \\ Qingqiang Kong, ${ }^{\text {ac }}$ Guohua Sun, ${ }^{a}$ Aziz Ahmad, (iD ac Xiaoming Li, ${ }^{\text {a }}$ Zonglin Yi ${ }^{d}$ and \\ Chengmeng Chen (iD *ae
}

\begin{abstract}
Porous carbon materials are an attractive choice as electrode materials for supercapacitors due to their large specific surface area, high conductivity, stable chemical properties and low cost. In recent years, the electrochemical performance of porous carbon-based supercapacitors has gained considerable attention and has been greatly improved via the rational design of morphology/porous structure and surface properties. However, for these porous carbon electrodes, there remains a limited understanding of practical ion dynamics, charge storage mechanisms and their influence on the electrochemical performance. Therefore, an in-depth fundamental understanding of the charge storage mechanisms, transport pathways of electrons/ions and the electrochemically active sites is extremely important for further building efficient supercapacitors. Existing reviews in the literature mainly focus on the preparation and properties of porous carbon. Herein, we specifically summarize the state-of-the-art progress, from in situ characterization experiments and theoretical calculations to understand the ion/charge storage in porous carbon electrodes of supercapacitors, and further discuss the structure-activity relationships between doping species and electrochemical performances. Furthermore, the challenges and future development associated with supercapacitors in practical applications are included.
\end{abstract}

\section{Introduction}

The rapid depletion of non-renewable energy sources and serious environmental issues largely stimulate the utilization of clean and renewable energy. ${ }^{1-3}$ Supercapacitors, as an intermittent step during energy usage, have attracted wide attention due to their ultrafast charge-discharge rate, high power density, low maintenance and long cycle life (Fig. 1). ${ }^{4-6}$ The energy storage mechanism and the upper limit of specific capacity of a supercapacitor is significantly dominated by the selected electrode. ${ }^{7-10}$ Therefore, a key target for an advanced supercapacitor is to select an ideal electrode material. Among various electrode materials, porous carbons are considered as an

\footnotetext{
${ }^{a}$ CAS Key Laboratory of Carbon Materials, Institute of Coal Chemistry, Chinese Academy of Sciences, Taiyuan, 030001, China. E-mail: ccm@sxicc.ac.cn

${ }^{b}$ Key Laboratory of Solidification Control and Digital Preparation Technology (Liaoning Province), School of Materials Science and Engineering, Dalian University of Technology, Dalian 116085, China

${ }^{c}$ University of Chinese Academy of Sciences, Beijing 100049, P. R. China

${ }^{d}$ School of Environment and Safety, Taiyuan University of Science and Technology, Taiyuan 030024, China

${ }^{e}$ Center of Materials Science and Optoelectronics Engineering,

University of Chinese Academy of Sciences, Beijing 100049, China
}

attractive electrode candidate for supercapacitors because of their large surface area, adjustable pore size, high conductivity and broad chemical stability. Currently, porous carbons prepared from biomass precursors such as coconut shells and wood have been used commercially. ${ }^{1,11,12}$ However, the limited energy density of the current supercapacitors highly prevents their widespread applications. The energy density of supercapacitors is critically dependent on the structure and properties of the porous carbon electrode, and therefore optimizing this is a key to enhance the electrochemical performances of supercapacitors.

In 2006, a major breakthrough was achieved by Gogotsi et al., who demonstrated that electrolyte ions could enter pores with sub-nanometer sizes. This enabled charge storage, resulting in a very high specific capacitance (Fig. 2). ${ }^{13}$ Since then, the origin of the anomalous increase in capacitance has received enormous attention. A dramatically increasing number of experimental and theoretical research studies have focused on understanding this phenomenon and designing new electrode materials with improved performances. ${ }^{14-19}$ Centeno et al. ${ }^{20,21}$ further proposed that the capacitance increase in carbon nanopores did not significantly depend on the micropore size but mainly on the surface available to the cation of the organic electrolyte. Furthermore, researchers demonstrated that the 


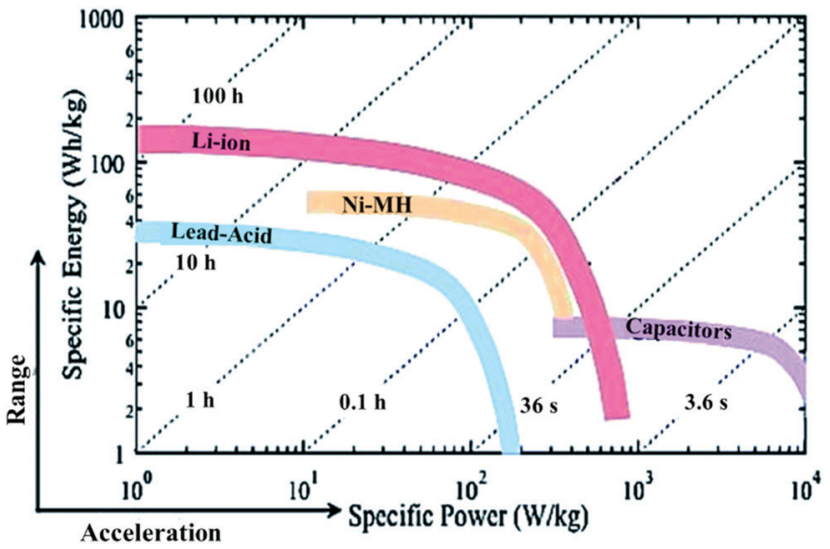

Fig. 1 Ragone plot comparing the specific energy density and specific power density of supercapacitors and other batteries. Reproduced with permission from ref. 4. Copyright 2015 American Chemical Society.

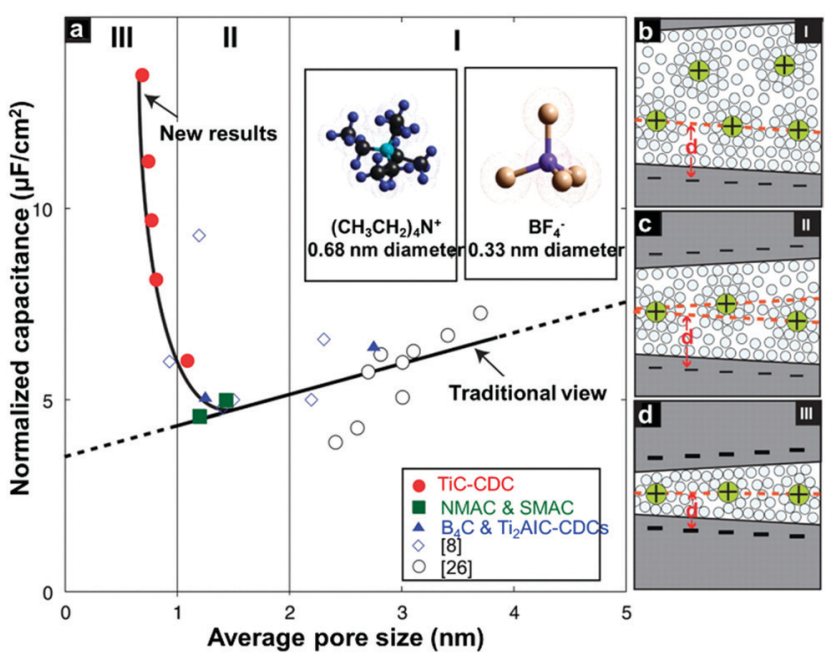

Fig. 2 (a) Plot of specific capacitance normalized by BET SSA and average pore size for carbons. Drawings of solvated ions residing in pores with the distance between adjacent pore walls: (b) greater than $2 \mathrm{~nm}$, (c) between 1 and $2 \mathrm{~nm}$, and (d) less than $1 \mathrm{~nm}$, to illustrate distinguishing behaviour schematically. Reproduced with permission from ref. 13. Copyright 2015 the American Association for the Advancement of Science.

confinement effect was responsible for the improved charge storage in nanopores through theoretical simulation and experimental studies. ${ }^{22-27}$ However, the confinement-induced effect causes a new problem in the form of large ion diffusion resistance within the micropores. ${ }^{12,28,29}$ In a recent report, Forse $e t a l .{ }^{4}$ presented a systematic study on the diffusion of $\mathrm{PEt}_{4}-\mathrm{BF}_{4} / \mathrm{AN}$ within the two commercial activated carbons of YP50 F and YP80 F by means of pulsed field gradient NMR. The results confirmed that the diffusivity of confined ions was two to three orders of magnitude smaller than the corresponding values obtained in the bulk solution. This led to undesired reductions of power density. Therefore, a strategy of enhancing the capacitive performance without power performance is greatly desired for the development of high performance supercapacitors. Inspired from natural phenomena, such as river deltas,
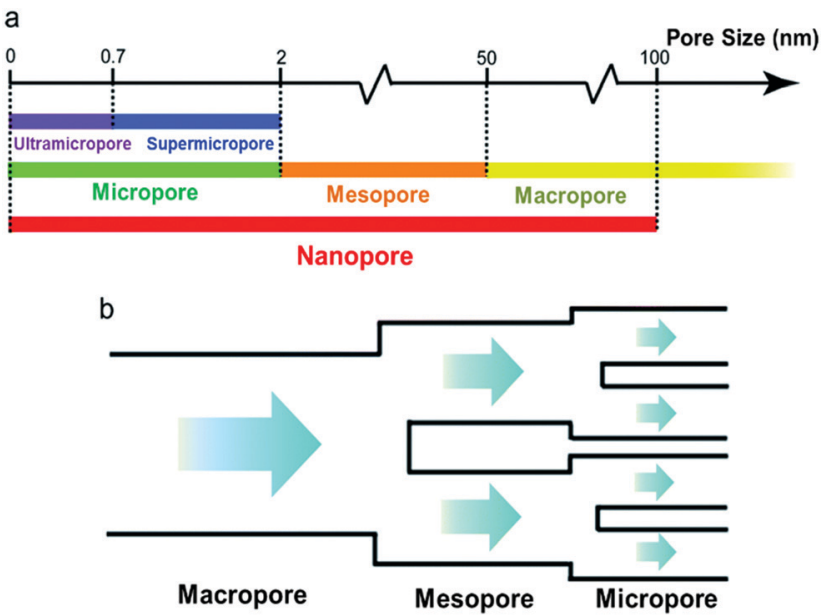

Fig. 3 (a) IUPAC classifications of pores based on the pore width. (b) Schematic illustration of an ion diffusion pattern in a typical hierarchical porous structure. Reproduced with permission from ref. 36. Copyright 2017 The Royal Society of Chemistry.

roots of trees and blood vessels, combining smaller and larger pores in a hierarchical manner is considered to be a reliable strategy to improve ion transport within electrode materials of supercapacitors. ${ }^{30,31}$ In this regard, a large number of hierarchical porous carbons, consisting of interconnected pores on different length scales from micro- $(<2 \mathrm{~nm})$ and meso$(2-50 \mathrm{~nm})$ to macropores $(>50 \mathrm{~nm})$, have been developed. ${ }^{32-35}$ The classifications of pore types are summarized in Fig. $3 \mathrm{a}^{36}$ The basic idea of using hierarchical macro-meso-microporous carbons is that small micropores with a high degree of geometric confinement can achieve a high energy density by efficiently screening the counterion charge, and macro- and mesopores can provide 'ion-highways' for the fast and efficient transport (Fig. 3b). ${ }^{37-39}$ Unexpectedly, Muslim Dvoyashkin et al. ${ }^{28}$ in their recent investigation revealed that the existence of a network of mesopores besides smaller micropores does not necessarily enhance ionic transport in carbon materials, as shown in Fig. 4.
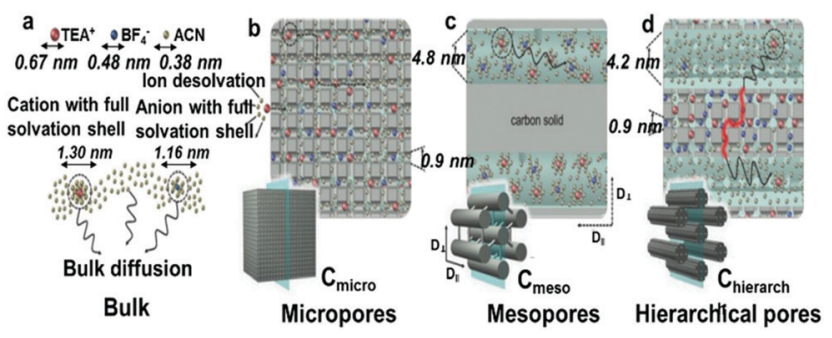

Fig. 4 Schematic representation of arrangement of different components of electrolyte solution-cations ( $\mathrm{TEA}^{+}$, red), anions $\left(\mathrm{BF}^{4-}\right.$, blue), and solvent (AN) (a) in the bulk solution, (b) in micropores of $\mathrm{C}_{\text {micro, }}$ (c) in mesopores of $C_{\text {meso, }}$ and (d) in the micro- and mesopores of the hierarchical $C_{\text {hierarch. }} D$ and $D$ denote diffusion coefficients measured in the directions parallel and perpendicular to the orientation of primary carbon rods. The red trajectory in (d) represents possible diffusion path within micropores of $\mathrm{C}_{\text {hierarch }}$ with ultimately low mobility. Reproduced with permission from ref. 31. Copyright 2018 Wiley-VCH Verlag GmbH \& Co. KGaA, Weinheim. 
This raises an important question about how to monitor the real electrochemical processes and thus effectively evaluate the optimization of the carbon architecture and pore size in porous carbon electrodes for supercapacitors. Recently, in situ characterization experiments, ${ }^{7,8,40,41}$ including in situ Infrared Spectroelectrochemistry, ${ }^{42}$ in situ $\mathrm{NMR},{ }^{11,43,44}$ in situ electrochemical quartz crystal microbalance measurements, ${ }^{45,46}$ and in situ X-ray transmission measurements, ${ }^{47,48}$ combined with advanced simulation techniques ${ }^{4-51}$ have been developed to track the real-time electrochemical processes, which provide insight into the microscopic mechanism underlying charge storage in the nanopores of supercapacitor electrodes. Consequently, there is a need to timely summarize the recent progress in the understanding of charge mechanisms in porous carbonbased electrodes, which will be helpful for further guiding the optimal design of advanced supercapacitor electrodes.

Heteroatom doping is another effective strategy to modify the carbon structure, as well as to enhance the electronic conductivity and wettability of carbon-based electrodes, thus improving their electrochemical performance. ${ }^{52-54}$ Many recent experimental and computational studies have already confirmed that heteroatom doping within the carbon skeleton can induce higher charge delocalization and density of donor states near the Fermi level, expand the interlayer spacing and enhance the wettability of the active material, which are highly desirable for high performance electrode materials. ${ }^{55-58}$ In general, $\mathrm{O},{ }^{59-61} \mathrm{~N},{ }^{56,62,63} \mathrm{P}^{64,65}$ and $\mathrm{B}^{66,67}$ are the most widely studied dopants for carbon-based materials. As different heteroatoms have different sizes and electro-negativities, their effects on tuning the properties of carbon materials may vary. ${ }^{54,68}$ Typically, oxygen doping within the carbon matrix can effectively modify the surface wettability and provide more active sites for charge storage. ${ }^{61}$ For nitrogen doping, it has been proven that the conjugation of the lone-pair electrons of $\mathrm{N}$ and the graphitic $\pi$-bonds of carbon materials can distort the carbon structure to create defects and available active sites. ${ }^{62}$ Compared with nitrogen, phosphorus has a larger atomic radius. Thus, P-doped carbon materials often show improved electron donating ability, better electron delocalization, and higher density of defect sites, which are highly desired for supercapacitors. ${ }^{64,65}$ Boron, acting as an electron acceptor owing to its three valence electrons, can enter the carbon lattice by substituting carbon, leading to a shift of the Fermi level to the conduction band, and further modulate the electronic structure of carbon materials. ${ }^{66,69}$ Hence, boron doped carbon materials display an enhanced capacitive performance in supercapacitors. Recently, dual and multiple heteroatom doping in comparison with sole-heteroatom doping has further optimized the nano/microstructure of carbon electrodes, which exhibit a range of unique properties due to the synergistic effect of multiple-element doping, thus greatly boosting the electrochemical performances. ${ }^{67,70-72}$ Here, the preparation and electrochemical performances of carbon materials doped with one and several heteroatoms for supercapacitors are briefly introduced. The configuration and functional mechanisms of dopant species in the carbon skeleton will be discussed in detail. It is hoped that this review can help readers to get a comprehensive picture on how to optimize heteroatom-doped carbon materials towards advanced supercapacitors.

\section{Investigating charge-storage mechanisms of porous carbon by in situ characterization}

It has been commonly believed that the charge storage ability of supercapacitor electrodes arises from the electrostatic attraction between the ions of an electrolyte and the electronic charges stored on the electrode surface, which enables the formation of oppositely charged layers at the electrolyte/ electrode interface. ${ }^{1,73,74}$ Currently, the vast majority of supercapacitors mainly uses porous carbon with a large specific surface area as electrode materials (Fig. 6a), thus providing the high surface area available for ion adsorption. Initially, the Helmholtz model is used to describe the charge separation at the electrolyte/electrode interface. ${ }^{75,76}$ When an electrode of surface area $A\left(\mathrm{~m}^{2}\right)$ is polarized, the oppositely charged ions diffuse through the electrolyte to form a condensed layer with a thickness of a few nanometers in a plane parallel to the electrode surface ensuring charge neutrality. The accumulation of charges is called an electrochemical double layer. In this model, the potential in the vicinity of the electrode decreases as the distance $d(\mathrm{~m})$ between the ion and the electrode increases. The capacitance of the electrochemical double layer can therefore be defined by eqn (1):

$$
C=\frac{\varepsilon_{\mathrm{r}} \varepsilon_{0} A}{d}
$$

where $\varepsilon_{0}$ is the vacuum permittivity, $\varepsilon_{\mathrm{r}}$ is the relative permittivity of the electrolyte, and $d$ is the effective thickness of the double layer. Here, $C$ is linear with $A$. In view of the high specific surface area of porous carbon electrodes, the specific capacitance of a porous carbon-based supercapacitor will be much higher than that of a flat plate capacitor (Fig. 6b). ${ }^{77}$ However, the Helmholtz model does not consider some key practical factors such as the diffusion of ions in the solution and the interaction between the dipole moment of the solvent and the electrode (Fig. $5 \mathrm{c}$ and d). Thus these quantities (except for $\varepsilon_{0}$ ) are not very well understood for the interfaces derived from highly disordered materials. ${ }^{1}$ Many experimental phenomena cannot be well explained by only the Helmholtz model. After that, theoretical studies based on molecular dynamics and mean-field theories have shown ion-ion interactions and ion-carbon interactions and the influence of electrode pore size on the rates of ionic diffusion in supercapacitor electrodes. ${ }^{51,78-81}$ Nevertheless, theoretical studies certainly rely on assumptions and simplifications, which fall short in quantifying the local position of ions in complexly shaped pores and ions' in-pore motion. ${ }^{11,41,82}$ Recently, based on NMR studies of supercapacitors with porous carbon electrodes and organic electrolytes, three possible pathways for charge storage mechanisms are proposed including ion adsorption of counter-ions into carbon pores, desorption of co-ions from 
a Conventional capacitor
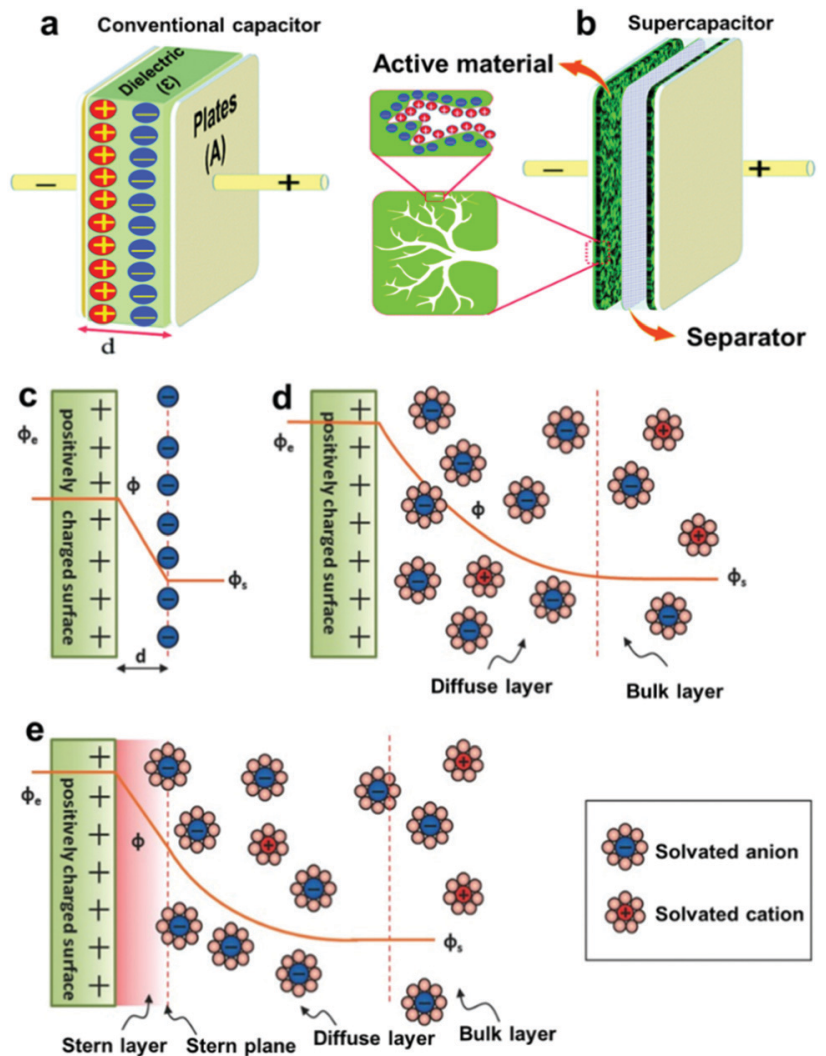

Fig. 5 Schematic representation of (a) a conventional capacitor consisting of two parallel plates separated by a thin insulating material and (b) a supercapacitor consisting of two porous electrodes separated by an ionpermeable separator and an electrolyte that ionically connects the two electrodes. Reproduced with permission from ref. 74. Copyright 2019 The Royal Society of Chemistry. (c) Helmholtz, (d) Gouy-Chapman, and (e) Stern model of the electrical double-layer formed at a positively charged electrode in an aqueous electrolyte. The electrical potential, $\phi$, decreases when transitioning from the electrode, $\phi_{\mathrm{e}}$, to the bulk electrolyte infinitely away from the electrode surface, $\phi_{\mathrm{s}}$. The Stern plane marks the distance of the closest approach of the ions to the charged surface. Note the absence of charges/ions in the Stern layer. The diffuse layer starts in the range of 10-100 nm from the electrode surface. Reproduced with permission from ref. 1. Copyright 2014 Wiley-VCH Verlag GmbH \& Co. KGaA, Weinheim.

carbon pores, or simultaneous adsorption of counter-ions and desorption of co-ions (Fig. 6) ${ }^{8,83}$ However, it is not clear that whether charging is a purely adsorptive process, or whether exchange of one set of ions by those with opposite charge and co-ion expulsion from the charged electrodes contribute to the formation of the electrical double layer. ${ }^{43,44,84}$

Considering the complex nature of charging in porous carbon electrodes of supercapacitors, it is urgent to develop novel in situ characterization methods to monitor electrolyte ion diffusion in the nanopores of supercapacitor electrodes. Thus, a bridge linking the macroscopic electrochemical performance and microscopic architectures of the electrode can be well built up. This could provide us with an in-depth understanding of the real electrochemical process of porous carbonbased supercapacitors and help scientifically develop further optimization strategies. Recently, a large number of studies

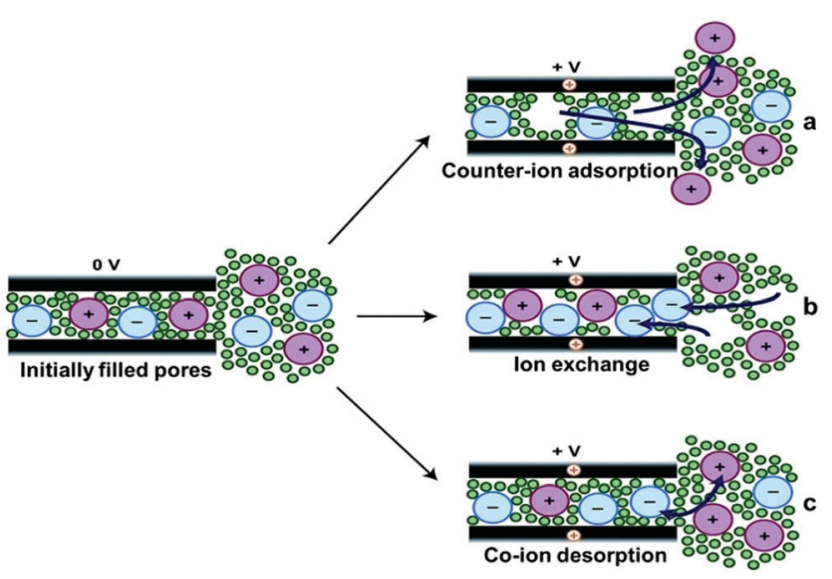

Fig. 6 Schematic illustrations of possible charge storage mechanisms for carbon pores that are wetted at zero potential. If the electrode surface is charged to a positive potential, an equal negative ionic charge can arise through either (a) the expulsion of cations from the pores, (b) adsorption of ex-pore anions into the pores, or (c) exchange of ex-pore anions for in-pore cations. Reproduced with permission from ref. 83. Copyright 2014 The Royal Society of Chemistry.

have testified the potential of an in situ electrochemical quartz crystal microbalance (EQCM) to track mass changes in porous carbon electrodes upon the charging process. ${ }^{45,46}$ Deviations from theoretical mass changes have been explained in terms of distinct charging regimes, which result from compositional differences in the ionic part of the electrical double layer. Fig. 7a shows a schematic of an EQCM set-up; the change of the quartz resonance frequency reflects the change of weight of the active material during the polarization. ${ }^{40}$ Fig. $7 \mathrm{~b}$ shows typical changes in the amount of cations and anions as a function of the charge accumulated in a porous carbon electrode during polarization in an organic electrolyte with different salts dissolved in PC. In this example, from the associated mass change and the comparison with the theoretical mass change
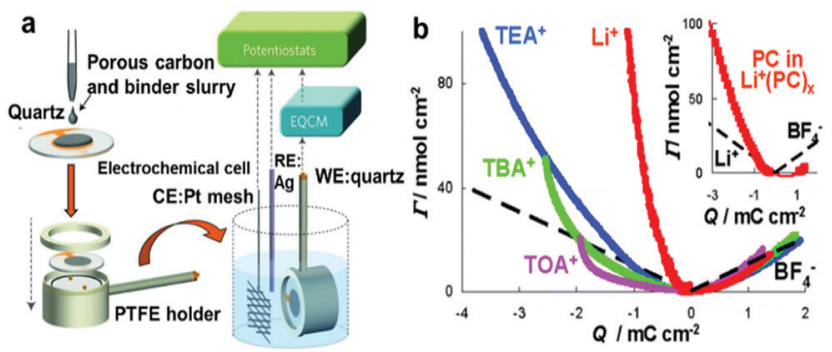

Fig. 7 (a) EQCM set-up. RE, reference electrode; $C E$, counter electrode; WE, working electrode. Reproduced with permission from ref. 37. Copyright 2016 Springer Nature. (b) Variation of the adsorption of ions with respect to the accumulated charge per unit area in aqueous electrolytes with various cations. The theoretical $\Gamma$ versus $Q$ plots (the broken black straight lines) were calculated on the basis of the Faraday law. The inset compares the amount of solvent molecules (the solid red curve), accompanying adsorption of the $\mathrm{Li}^{+}$cations (the left broken black line). Their ratio is close to 4 , confirming the partial desolvation of $\mathrm{Li}^{+}$cations confined in carbon micropores. Reproduced with permission from ref. 42. Copyright 2010 American Chemical Society. 
of bare ions calculated on the basis of the Faraday law, there was a gradual increase of confinement of the cations in the accessible AC micropores in the order: $\mathrm{Li}^{+}<\mathrm{TEA}^{+}<\mathrm{TBA}^{+}<$ $\mathrm{TOA}^{+}$. This implies that the bulkier cations are less effective (as the counterions) in keeping the electroneutrality in the carbon pores during their charging, forcing the smaller (and hence more mobile) co-ions to play an increasing role in the charge compensation mechanism. ${ }^{45}$ Levi and co-workers further used the in situ EQCM to study metal ion adsorption in porous carbons. They mentioned that the adsorbed $\mathrm{Li}^{+}, \mathrm{Na}^{+}$, $\mathrm{K}^{+}$and $\mathrm{Cs}^{+}$ions are tightly attached to $2.0,0.8,0.1$ and 0.0 water molecules on average. The confinement of the ions is therefore accompanied by a substantial decrease in their solvation number (number of solvent molecules around the ion), thus leading to an enhanced capacitance in subnanometer size carbon pores. ${ }^{85}$ This method has also been used in ionic liquids to characterize ion adsorption in carbide-derived carbon with large $(1 \mathrm{~nm})$ and small $(0.65 \mathrm{~nm})$ pore sizes (Fig. $8 \mathrm{a}-\mathrm{f})$. It was found that there were less solvated acetonitrile molecules in $0.65 \mathrm{~nm}$ pores than in $1 \mathrm{~nm}$ pores, indicating that the ion desolvation occurs to be able to fit in the smaller pores.
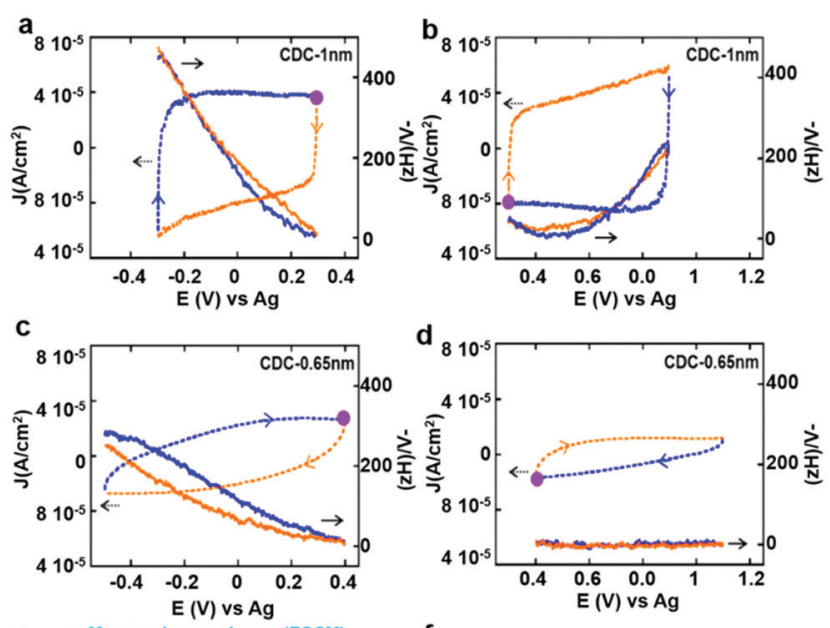

e - Measured mass change (EQCM)
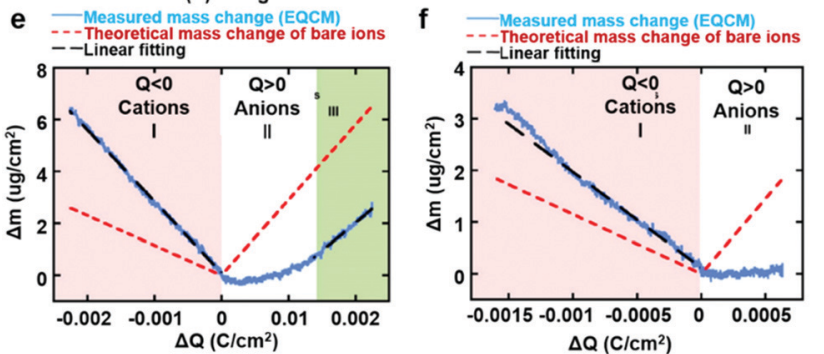

Fig. $8 \mathrm{CV}$ and EQCM frequency response of (a and b) CDC-1 nm and ( $c$ and d) CDC- $0.65 \mathrm{~nm}$ (e and f) in $2 \mathrm{M} \mathrm{EMI-TFSI/AN} \mathrm{below} \mathrm{(} \mathrm{a}$ and c) and above ( $b$ and $d$ ) the immersion potential (the purple dot is where the cycle starts; orange and blue curves indicate charge and discharge of ions, respectively). Electrode mass change vs. charge during the polarization of (e) CDC-1 nm and (f) CDC-0.65 nm in $2 \mathrm{M} \mathrm{EMI-TFSI} \mathrm{+} \mathrm{AN} \mathrm{(blue} \mathrm{solid} \mathrm{lines}$ are measured mass change (EQCM); red dashed lines are the theoretical mass change of neat ions calculated from Faraday's law and the black dashed line shows the linear fitting of measured mass change). Reproduced with permission from ref. 46. Copyright 2014 American Chemical Society.
These results clearly indicate that the partial desolvation of the ions occurs when accessing small pores. The confinement of the ions in narrow nanopores results in more efficient energy storage. $^{46}$

The morphological complexity of the pore network in a porous carbon electrode has been considered for the first time by Merlet et al. ${ }^{17,24}$ who have shown that knowledge of the exact positions of ions within the nanopores is essential, because all physical processes depend on the detailed interactions between ions, solvent molecules, and pore walls. In situ small-angle $\mathrm{X}$-ray scattering, as a well-established spectroscopic technique, has the features of low cost and easy operation to monitor ion positions and concentration changes during electrochemical operation. ${ }^{25,86,87}$ The results demonstrate that the initial fast charge neutralization in a porous electrode leads to a nonequilibrium ion configuration. Subsequently, charge-neutral equilibration slowly increases the ion concentration towards counter-ion adsorption. With the combination of in situ smallangle X-ray scattering and atomistic modelling, it has been shown that in addition to concentration changes, there is a local ion rearrangement across the nanopores combined with partial desolvation (Fig. 9). Actually, the ion rearrangement greatly optimizes the screen repulsive interactions between counter-ions, resulting in preferentially occupying sites with the highest possible degree of confinement. This mechanism naturally explains the often reported increase of surfacenormalized capacitance with decreasing micropore size. ${ }^{13,19}$ Infrared spectroscopy (IR) is a type of absorption spectroscopy, which has the advantages of rapid sample analysis, suitability for a wide variety of analytes, easy operation and so on. Yossef A. Elabd et al. employed in situ infrared spectroelectrochemistry for the first time to directly measure the ion dynamics of EMIm-TFSI in electrodes made of porous nanosized carbidederived carbons (CDCs) and nonporous onion-like carbons (OLCs) as a function of voltage (Fig. 10). This provides direct experimental evidence of cations and anions of EMIm-TFSI

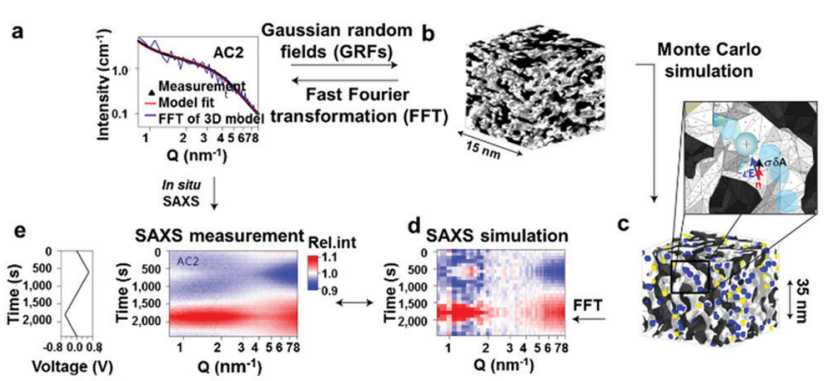

Fig. 9 (a) SAXS intensity versus scattering vector length $(Q)$ of measured data (black) for sample AC2 in vacuum and an accurate model fit (red). (b) Resulting real-space pore structure (here: $15 \times 15 \times 15 \mathrm{~nm}^{3}$ ) using the concept of Gaussian random fields (GRFs). (c) The pore structure (here: 3D subvolume with $7 \times 7 \times 7 \mathrm{~nm}^{3}$ ) is populated with cations (blue) and anions (yellow) in a homogeneous dielectric water phase with the ion concentration obtained from X-ray transmission. (d) Simulated relative SAXS curves are compared to the measured relative SAXS intensities with the applied voltage signal (e). Reproduced with permission from ref. 25. Copyright 2017, Springer Nature. 
a
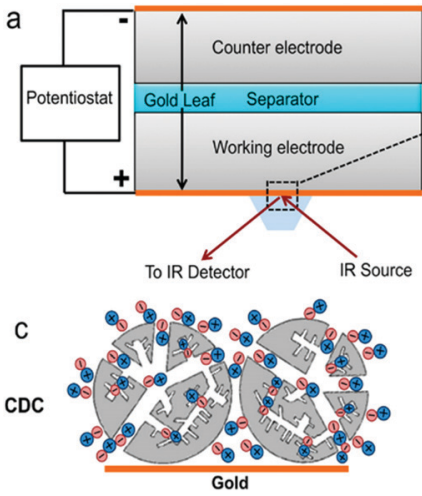

d

OLC

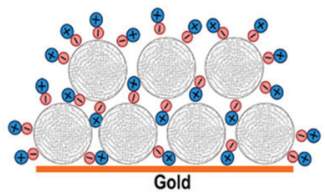

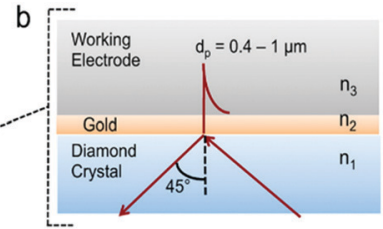

e

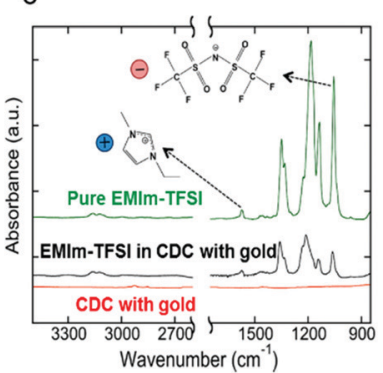

Fig. 10 (a) Schematic of the spectroelectrochemical experiment with the supercapacitor on the ATR crystal of the FTIR spectrometer and (b) magnified view of the crystal/electrode interface showing the sampling depth into the working electrode in the range of $0.4-1 \mu \mathrm{m}$. Illustration of ions in electrodes consisting of (c) porous CDC particles and (d) exohedral OLC particles on a gold current collector. (e) Infrared spectra of pure EMIm-TFSI (no gold) and CDC on gold with and without EMIm-TFSI. Reproduced with permission from ref. 42. Copyright 2013 American Chemical Society.

entering and exiting CDC pores during charging/discharging and the ability of the organic solvent to enter and exit the nanoporous was ionic liquid (IL) concentration dependent. ${ }^{42}$ These in situ methods reveal distinct dependencies of the ion/charge storage mechanism on the electrolyte salt concentration, pore size and the nature of the used ions. However, the in-pore ion motion cannot be directly detected only by the above-mentioned techniques, and so a full description of the ion/charge storage mechanism has not been achieved.

Actually, seeing what is happening inside the pores of porous carbon during charging is quite important for understanding well the charge storage mechanisms in supercapacitors. In particular, selective observation and quantification of anions and cations within the highly complex porous structure are highly desired, which can be used to distinguish between the different possible charging mechanisms. ${ }^{44,73}$ In this regard, solid-state NMR spectroscopy stands out as a highly localized and element-selective probe of chemical environments. The changes in intensities of the in-pore resonance provide a quantitative measure for the number of in-pore species in the porous carbon electrode. In situ NMR study on typical YP50 F activated carbon soaked with $\mathrm{NEt}_{4}-\mathrm{BF}_{4} / \mathrm{AN}$ revealed the change in the intensity and the nucleus independent chemical shift of in-pore resonance at different charge states, identifying different charge storage regimes. ${ }^{73}$ Clare P. Grey and co-workers have investigated the adsorption properties of ions in organic electrolyte-based supercapacitors through a comparison of the in-pore resonance intensities with calibration samples. An increase in the in-pore resonance intensity during charging showed that the
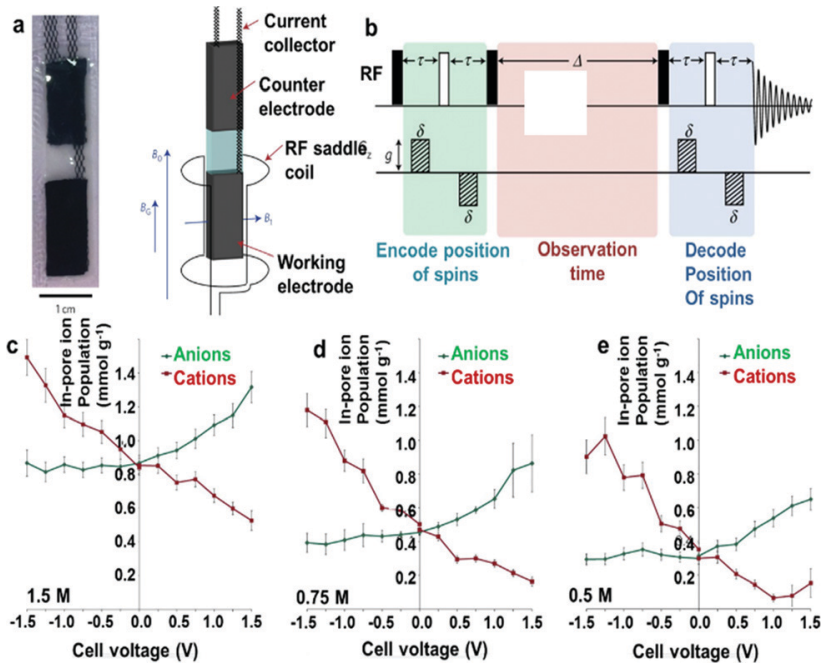

Fig. 11 Introducing pulsed field gradient NMR of supercapacitors. (a) A photo of a pouch cell and a schematic of the in situ pulsed field gradient (PFG) set-up showing the orientation of the magnetic field. (b) 13-interval sequence used for PFG NMR experiments; radiofrequency and magnetic field gradient $(\mathrm{Gz})$ pulses are shown. Reproduced with permission from ref. 4. Copyright 2017 Springer Nature. (c) In-pore ion populations of supercapacitor electrodes for electrolyte concentrations of (c) $1.5 \mathrm{M}$, (d) $0.75 \mathrm{M}$ and (e) $0.5 \mathrm{M}$ at different states of charge in the range $-1.5 \mathrm{~V}$ to $1.5 \mathrm{~V}$. Reproduced with permission from ref. 43. Copyright 2015 Springer Nature.

studied ions were adsorbed into the micropores, and conversely, ion expulsion. ${ }^{43}$ The combination of in situ NMR and EQCM measurements distinguished the different possible charging mechanisms over the potential range studied, which enabled a detailed investigation of the double-layer structure as a function of the applied cell voltage (Fig. 11). At a cell voltage of $0 \mathrm{~V}$, equal numbers of anions and cations were densely packed inside the micropores of the porous carbon electrode. Upon charging for the negative potentials, the electronic charge stored on the electrode surface was balanced by the absorption of cations into the micropores. For positive potentials, the stored electronic charge was balanced by the absorption of anions into the micropores and simultaneous expulsion of cations. The asymmetry in ion sizes may be one reason for the different mechanisms in the two charging regimes. They also showed that the electrolyte concentration and nanopore size had significant effects on in-pore diffusion, and on the exchange of ions between bulk and in-pore sites. Ghenima Oukali and co-workers further used in situ NMR to study the effects of the carbon texture at the nanometer scale on the ion flux and charging mechanism by comparing a conventional nanoporous carbon and a carbide-derived carbon. ${ }^{88}$ It was shown that the large proportion of small micropores led to a more symmetric behaviour of cations and anions during the charge/ discharge process and an increased capacitance. However, large ion transport resistance within the small micropores can lead to the decrease of power density. By comparing the ${ }^{13} \mathrm{C}$ NMR spectrum of the microporous carbon electrode and the mesoporous carbon electrode with an ionic mixture electrolyte before and after charging, an in-cell selective charging behavior has been 

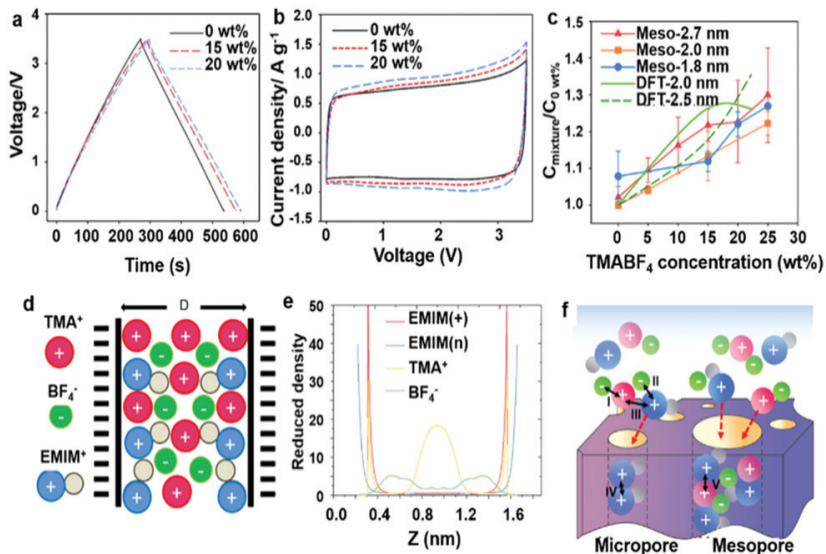

Fig. 12 (a) GCD at $1 \mathrm{~A} \mathrm{~g}^{-1}$ and (b) $\mathrm{CV}$ curves at $10 \mathrm{mV} \mathrm{s}$ of the mesopore-rich carbon meso-2.0 nm. (c) Relation between normalized capacitance and $\mathrm{TMABF}_{4}$ concentration with three mesopore-rich carbon materials. (d) Schematic representation of a room-temperature ionic mixture in a charged slit pore. (e) Distribution of cations and anions inside a slit pore (pore size $=1.875 \mathrm{~nm})$ with negatively charged walls $(\psi-\mathrm{PZC}=$ $-1.5 \mathrm{~V}$ ) for the case of $22.2 \mathrm{wt} \% \mathrm{TMABF}_{4}$ in $\mathrm{EMIMBF}_{4}$. (f) Schematic of the ionic interaction induced selective charging behavior of ionic mixture electrolyte. Black arrows represent ionic interactions: (I) $\mathrm{TMA}^{+}-\mathrm{BF}_{4}{ }^{-}$ interaction; (II) $\mathrm{EMIM}^{+}-\mathrm{BF}_{4}{ }^{-}$interaction; (III) steric effect near high confinement pore; (IV) $\mathrm{EMIM}^{+}-\mathrm{EMIM}^{+}$interaction and (V) $\mathrm{TMA}^{+}-\mathrm{EMIM}^{+}$interaction; red arrows $(\rightarrow)$ represent ion diffusion. Reproduced with permission from ref. 89. Copyright 2017 American Chemical Society.

demonstrated through balancing the interactions between charge carrying ions of the electrolyte and the interaction of the electrolyte within mesopore-rich carbon (Fig. 12). By using this method, the energy density of the supercapacitor was increased without sacrificing the power density. ${ }^{89}$ The above results have confirmed that the numbers of anions/cations within the porous carbon electrode changed as a voltage was applied. Importantly, the charge storage mechanism was sensitive to the used electrolyte and its concentration. So controlling the charge storage mechanisms through appropriate combination of ions and solvents as well as electrode structures may be a useful strategy to tune the energy and power performances of supercapacitors for a range of different applications.

\section{Insight into the activity promotion effect of doping species in the carbon matrix by in situ characterization combined with theoretical methods}

The above mentioned charge storage mechanisms are helpful to understand well the process of charge storage inside porous carbon electrodes and provide practical design principle for the development of more efficient supercapacitors. Furthermore, various heteroatom dopants such as $\mathrm{O}, \mathrm{N}, \mathrm{P}$ and $\mathrm{B}$ can induce structural distortions and charge-density as well as electronicstructure modulations in porous carbon materials due to their different size and electronegativities, which is useful for energy storage. ${ }^{68,90,91}$ Recently, a range of heteroatom mono- or
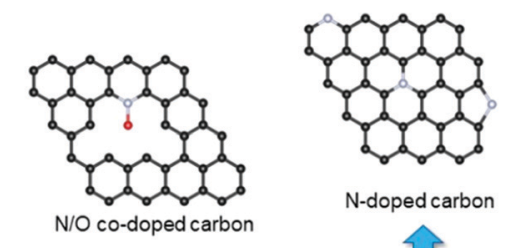

$\mathrm{N}$-doped carbon

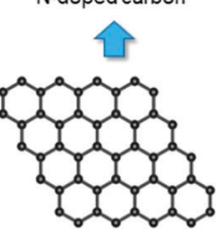

Carbon
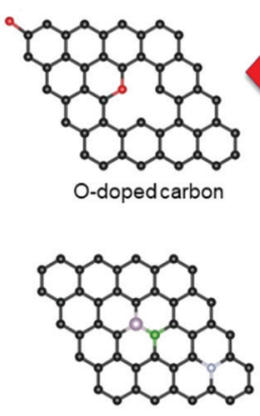

Triple-element doped carbon

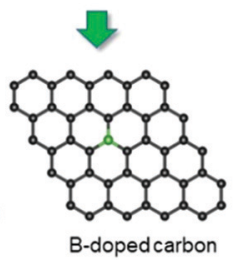

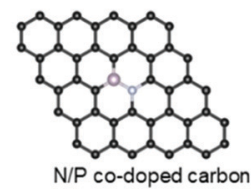
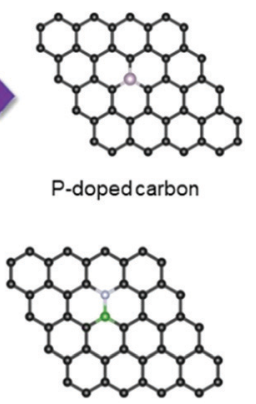

N/B co-doped carbon
Fig. 13 Schematic illustration of the structures of various heteroatomdoped carbons.

multi-doped porous carbon materials have been widely reported to show improved capacitive behavior in supercapacitors. Further in situ characterization and computational studies have been used to explain the configuration optimization and functional mechanisms of dopant species in the carbon matrix. Herein, we will summarize the latest advances in the configuration optimization and functional mechanisms of dopant species in the carbon skeleton (as shown in Fig. 13) and help readers to get a fundamental understanding of the physical-chemical nature of the activity promotion effect caused by heteroatom doping.

\subsection{O-Doped porous carbons}

Oxygen-containing groups are the most common functional groups in porous carbons, which can be introduced through a residue from the carbon sources, various post-treatment methods and an oxidizing environment. The introduced oxygen functional groups can not only improve the surface wettability and reversible pseudocapacitive reactions but also influence the charge or mass transfer. ${ }^{91,92}$ Many studies have optimized the content and species of oxygen functional groups on carbonbased electrodes to strengthen the electrochemical properties. Stoeckli $e t$ al. reported that the KOH-treatment of commercial activated carbon led to highly pure carbons with effective surface areas and improved surface chemistry. They further concluded that the redox reactions of quinone-hydroquinone functional groups at the carbon interface can be ascribed to the pseudocapacitance behaviour in aqueous electrolytes. ${ }^{93}$ Conway et al. also considered that the quinone or aldehyde functional groups underwent a redox reaction, contributing to the overall capacitance of the carbon electrode in an aqueous electrolyte. ${ }^{92}$ Combined with the temperature programmed desorption (TPD) method, it can be learned that the surface oxygen species were classified into two groups including the functional groups desorbed as $\mathrm{CO}$ (CO-desorbing complexes) 
and those desorbed as $\mathrm{CO}_{2}\left(\mathrm{CO}_{2}\right.$-desorbing complexes). $\mathrm{CO}$ was mainly derived from hydroxyl, carbonyl and quinone groups, while $\mathrm{CO}_{2}$ was mainly from carboxyl, anhydride and lactone groups. The groups converted into $\mathrm{CO}$ offered a larger capacitance than those converted into $\mathrm{CO}_{2} \cdot{ }^{94-97}$ Hirokazu Oda's team accurately compared the contribution of phenolic hydroxyl groups (producing $\mathrm{CO}$ ) and carboxyl groups (producing $\mathrm{CO}_{2}$ ) to the capacitance by using the XPS test. Experimental results showed that the growth rate of the capacitance became more sensitive to the number of phenolic hydroxyl groups with the increase in the number of these two groups. ${ }^{98}$ Yaohui Zhang et al. systematically analyzed the reasons for the increase of the capacity resulting from oxygen functional groups and the mechanism through which the pseudocapacitance was provided by the oxygen functional groups in the acid/alkaline aqueous electrolyte. The results showed that the Faraday pseudocapacitance was contributed by the electron transfer or redox reaction. Specifically, in the acidic aqueous electrolyte, the electrons on the oxygen functional groups were attracted by $\mathrm{H}_{3} \mathrm{O}^{+}$, which led to the separation of positive and negative charges, thus making the redox reaction possible. While in the alkaline aqueous electrolyte, the pseudocapacitance might be caused by the insertion/deinsertion reaction of the hydrated ions in the pore..$^{94}$ Recently, Wang et al. fabricated oxygendoped carbon cloth by annealing commercial carbon cloth in air at low temperatures. In order to understand the capacitive behaviors, the correlation of the faradaic redox reaction with the configurations of oxygen surface groups was carefully investigated. The results showed that pseudocapacitance could be enhanced by fixing oxygen functional groups on micropores rather than on plane carbon..$^{92}$ In another example, it has been demonstrated that the introduction of surface oxygen groups can alter the capacitive mechanism and contribution of carbonbased electrodes, especially for aqueous electrolytes. In order to efficiently preserve the micro-meso-macroporous structures from the original sample, a mild $\mathrm{HNO}_{3}$ hydrothermal oxidation has been used to finely tailor the degree and a variety of oxygen surface groups in the hierarchical porous carbon, as shown in Fig. 14a. By de-convoluting the redox peaks of cyclic voltammetry curves, it was found that the surface oxygen species not only slightly enhanced the surface-controlled capacitance including EDLC and pseudocapacitance, but also dramatically improved the diffusion-controlled capacitance through potassium and hydroxyl ion insertion/deinsertion into the underutilized micropores. However, the stronger oxidation of the carbon spheres can cause a rapid decline of the electrochemical performance due to the blocking of electrolyte ion diffusion by a large amount of oxygen (Fig. 14b-j). Therefore, an unreasonable increase in the proportion of oxygen functional groups will tightly trap ions and reduce the probability of ion intercalation. ${ }^{99}$ Compared with aqueous electrolytes, organic electrolytes provide a broader electrochemical stability window $(>2 \mathrm{~V})$, which can significantly boost the energy densities of electric double-layer capacitors. ${ }^{100}$ At present, the majority of commercial electric double-layer capacitors utilize organic electrolytes. ${ }^{101}$ Unfortunately, the surface oxygen groups of carbon electrodes in
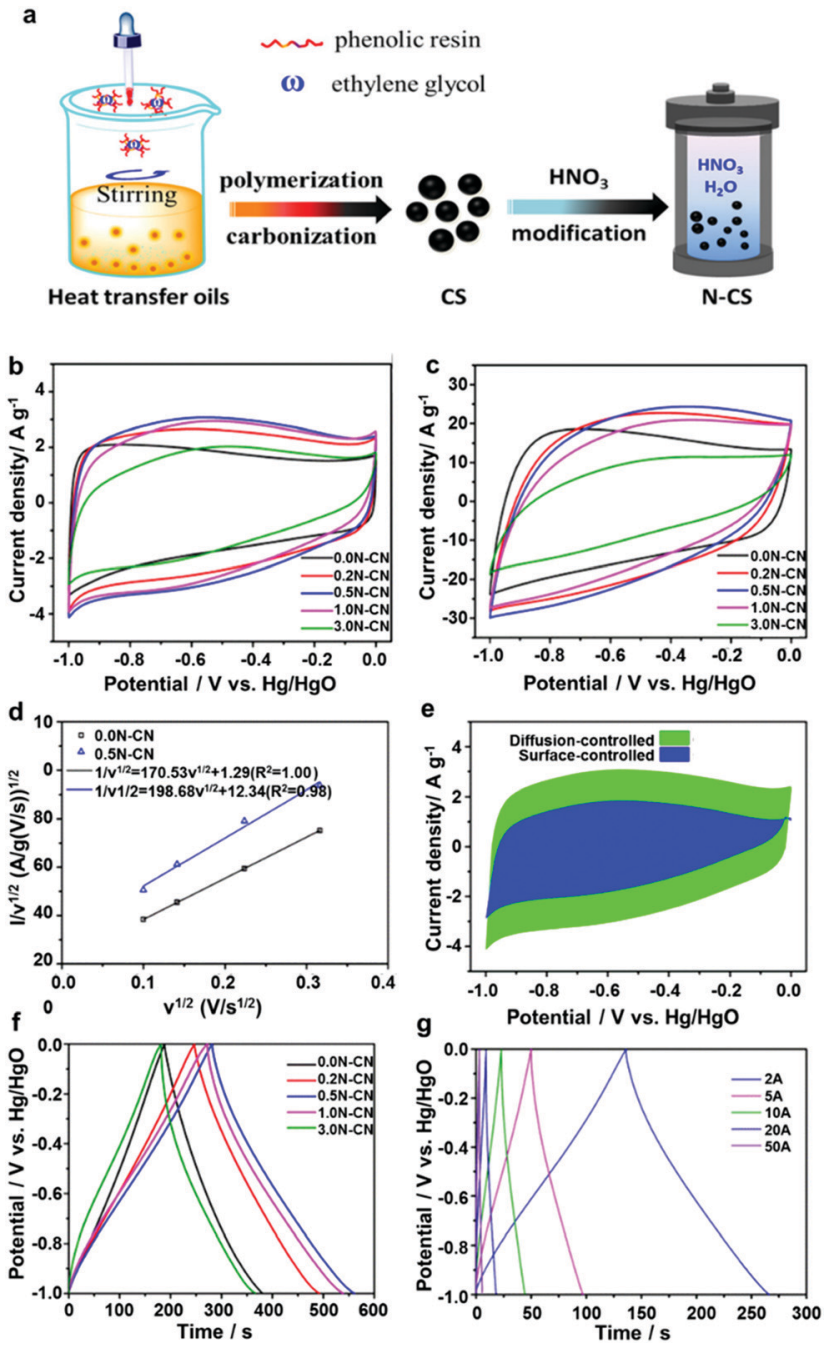

Fig. 14 (a) Schematic illustration of the fabrication of N-doping carbon microspheres from carbon microspheres. CV curves of all the samples at (b) $10 \mathrm{mV} \mathrm{s}^{-1}$ and (c) $100 \mathrm{mV} \mathrm{s}^{-1}$. (d) Relationship between $i(\nu) / \nu^{1 / 2}$ and $\nu^{1 / 2}$. (e) Surface-controlled (blue) and diffusion-controlled (green) contribution to a current density of $0.5 \mathrm{~N}-\mathrm{CS}$ at $10 \mathrm{mV} \mathrm{s}{ }^{-1}$. (f) GCD curves of all the samples at $1 \mathrm{~A} \mathrm{~g}^{-1}$. (g) Grain size distribution curves of $0.5 \mathrm{~N}-\mathrm{CS}$ from 2 to $50 \mathrm{~A} \mathrm{~g}^{-1}$. Reproduced with permission from ref. 99. Copyright 2019 American Chemical Society.

organic electrolytes tend to be decomposed and block a part of porosity, causing rapid aging of the capacitor. ${ }^{102,103}$ Recently, we reported the optimization of the oxygen-containing functional groups in the carbon skeleton by hydrogen thermal reduction, resulting in the stabilized electrochemical interface between the electrode and organic electrolyte. The structural evolution of the oxygen-containing functional groups in the carbon lattice during the hydrogen thermal reduction is systematically studied by TPR-MS, XPS, Boehm titration coupled with the assistance of density functional theory (DFT) calculations. ${ }^{101,103}$ The structure-activity relationships between oxygen configurations and electrochemical performances are also established. The results demonstrated that both of $\mathrm{C}=\mathrm{O}$ and $\mathrm{C}-\mathrm{O}-\mathrm{C}$ configurations are the most stable structure in the carbon lattice, which is beneficial for the stability of the electrochemical interface. Thus, the optimized 
carbon electrode exhibits an ultralow self-discharge and leak current as well as improved cycling stability in organic electrolytes. Therefore, regardless of whether in aqueous electrolytes or organic electrolytes, the rational optimization of oxygen configurations in carbon materials is an effective strategy to satisfy a broad range of practical applications.

\subsection{N-Doped porous carbons}

Nitrogen, one of the neighbour elements to carbon in the same period, is the most studied doping atom. Nitrogen has an atomic radius similar to that of carbon but has different electronegativity and electronic configuration. Thus, the introduction of nitrogen into the carbon electrode can greatly adjust the electronic properties while minimizing the lattice mismatch. ${ }^{104,105}$ Meanwhile, the conjugation of the lone-pair electrons of $\mathrm{N}$ and the graphitic $\pi$-bonds of carbon materials can further distort the carbon structure to create defects and available active sites. ${ }^{90}$ According to the bonding environments, four types of doped nitrogen can be found in $\mathrm{N}$-doped porous carbon, such as pyrrolic-N, pyridinic-N, quaternary-N/graphitic$\mathrm{N}$ and $\mathrm{N}$ oxides of pyridinic- $\mathrm{N}$, as shown in Fig. $15 .{ }^{106}$ Specifically, pyridinic- $\mathrm{N}$ is bonded with two $\mathrm{C}$ atoms as a member of hexagon, and pyrrolic- $\mathrm{N}$ with two $\mathrm{C}$ atoms by forming pentagon; both types of $\mathrm{N}$ occur at the edge of the layer but can also occur at the inside of the layer by associating with vacancies. Both pyridinic- $\mathrm{N}$ and pyrrolic- $\mathrm{N}$ have relatively high reactivity for the oxidation reduction reaction. For graphitic-N, the $\mathrm{N}$ atom substitutes for a $\mathrm{C}$ atom in the graphene hexagonal ring, which can promote the charge-transfer rate at the electrode/ electrolyte surface. Kunlin Liu et al. reported a bottom-up strategy to prepare $\mathrm{N}$-doped porous carbons by sodium metal-assisted carbonization of pyrrole. The results showed that the contact angle of samples gradually increased as the nitrogen content decreased, indicating that the introduction of $\mathrm{N}$ greatly improved the wettability of the carbons. This further reduced the interface

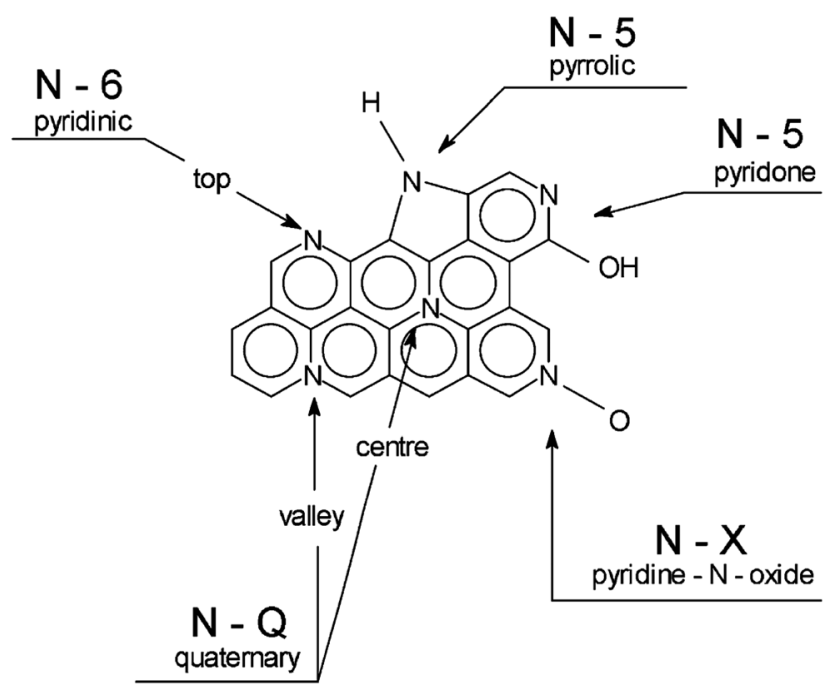

Fig. 15 Bonding configurations for nitrogen atoms in carbon materials. Reproduced with permission from ref. 106. Copyright (C) 2003 Elsevier Science Ltd. resistances of the electrode/interface and therefore enhanced the rate performance of the $\mathrm{N}$-doped porous carbon-based electrode. ${ }^{107}$ Dazhang Zhu et al. synthesized high-level N-doped porous carbons (N-MCSs) via a simple Schiff-base chemistry. As supercapacitor electrodes, N-MCSs delivered a high gravimetric capacitance, good rate capability and cycling stability. The excellent electrochemical performance could be attributed to well-developed supermicropores and functionalized groups with high nitrogen contents of $8.71 \%$. Importantly, the predominant $\mathrm{N}-5$ and N-6 were electroactive and showed an inclination towards electron donation to provide pseudocapacitance in $\mathrm{KOH}$ electrolyte. The presence of N-Q not only improved the electronic conductivity, but also provided additional defects for interacting with the anions in both acidic and basic electrolytes. ${ }^{108}$ Fei Sun et al. fabricated nitrogen-rich carbon particles by a continuous aerosol spraying method. The Raman spectrum of the NRC particles showed a high peak intensity ratio of the D to G band, indicating that there are a significant number of structural defects in the carbon framework due to $\mathrm{N}$ doping. The nitrogen-rich carbon (NRC) based supercapacitor can deliver an energy density of up to $50.6 \mathrm{~W} \mathrm{~h} \mathrm{~kg} \mathrm{k}^{-1}$ at a power density of $0.4 \mathrm{~kW} \mathrm{~kg}^{-1}$. Moreover, even at a high power density of $15.6 \mathrm{~kW} \mathrm{~kg}^{-1}$, the energy density remains high, at $19.5 \mathrm{~W} \mathrm{~h} \mathrm{~kg}^{-1}$. The excellent performance of NRC could benefit from the synergistic effect of large degree of N-doping, favorable pore structure and high surface area. Combined with density functional theory calculations, it can be concluded that pyridinic- $\mathrm{N}$ with lone pair electrons and pyrrolic- $\mathrm{N}$ with unpaired electrons could bond with $\mathrm{K}^{+}$ directly with the highest adsorption energies, which potentially provides more sites for ion adsorption and so improves the specific capacitance of EDLC. ${ }^{109}$ Hui-Ming Cheng et al. further proposed two mechanisms for the redox actions on nitrogendoped carbon electrodes in alkaline electrolytes based on an ex situ coupled CV-XPS study: (1) the double-electron-transfer association/dissociation of hydroxy groups between pyridone and pyridine; (2) single-electron-transfer conversions between pyridine and oxidized pyridine. ${ }^{110}$ However, the fabrication of non-doped and N-doped carbon materials with the same material characteristics such as porous structure, specific surface area and graphitic crystallinity is a very difficult work, which leads to the fact that the real contribution of various nitrogen functional groups to the capacitive performances in different electrolytes is still unclear experimentally. For instance, Titirici et al. prepared nitrogen and oxygen containing carbons by a hydrothermal method and subsequent $\mathrm{KOH}$ activation. They analyzed the supercapacitor performance of the as-obtained nitrogen-rich mesoporous carbons in different electrolytes. It was found that the capacitive performances of the obtained nitrogen-rich mesoporous carbons in an acidic electrolyte are better than in a basic electrolyte. They attributed this to the surface characteristics of those carbons. The higher the amount of nitrogen, the higher was the basicity of the carbon surface, resulting in better performance in the acidic electrolyte. ${ }^{111}$ Olejniczak et al. also found that the nitrogen doped mesoporous carbons derived from gelatin biomolecules displayed higher specific capacitance in $\mathrm{H}_{2} \mathrm{SO}_{4}$ than in $\mathrm{KOH}$ electrolyte. They concluded that the capacitive performance 

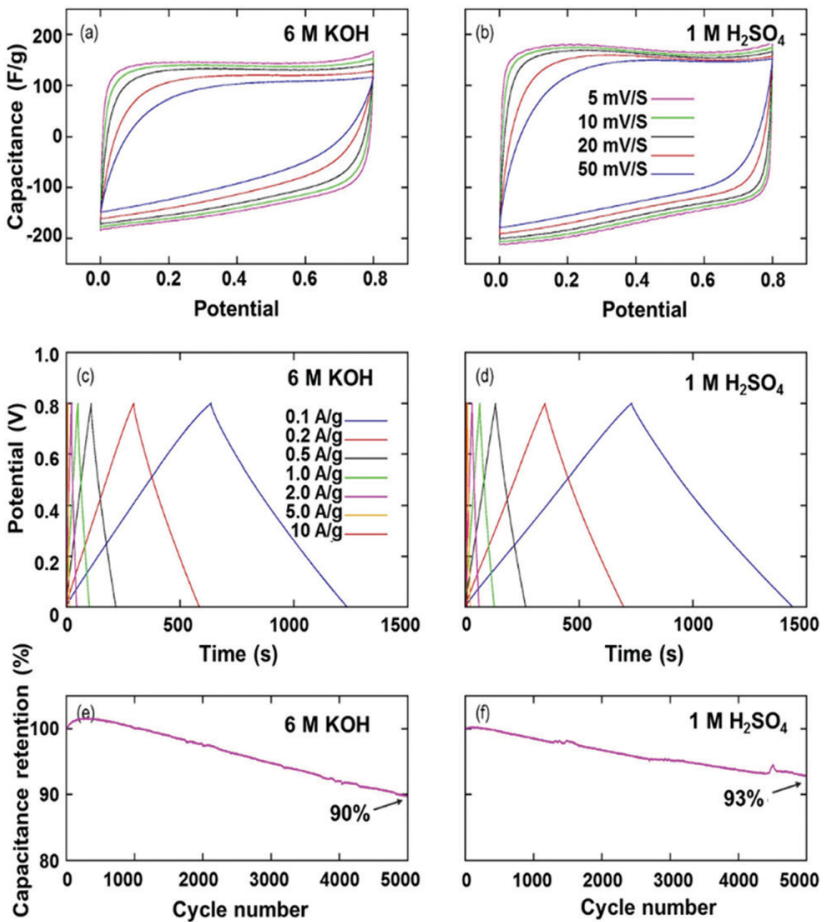

Fig. 16 The electrochemical capacitive performance (two-electrode configuration) of nitrogen-rich mesoporous carbons in $6 \mathrm{M} \mathrm{KOH}$ and $1 \mathrm{M} \mathrm{H}_{2} \mathrm{SO}_{4}$ aqueous solutions: (a and b) CVs recorded at various sweep rates; ( $c$ and d) galvanostatic charge/discharge curves recorded at various current densities; (e and f) cyclic durability at a current density of $2 \mathrm{~A} \mathrm{~g}^{-1}$. Reproduced with permission from ref. 112. Copyright 2015 Elsevier Ltd.

of the carbons was improved by virtue of the pseudocapacitance effect of nitrogen functional groups in the acidic solution, which was demonstrated by the shape of CV curves recorded in the acidic medium (Fig. 16). ${ }^{112}$ Contrary to the above-mentioned examples, some reports pointed out that nitrogen doped carbons showed higher specific capacitance in a basic electrolyte than in an acidic electrolyte. ${ }^{113-115}$ For example, Cazorla-Amorós et al. found that the pyridinic-N functional groups in NCNTs displayed different contributions to the specific capacitance in different electrolytes. In other words, the specific capacitance of nitrogenated carbon nanotubes (NCNTs) in $\mathrm{KOH}$ was much higher than that in $\mathrm{H}_{2} \mathrm{SO}_{4}$ electrolyte. The lower specific capacitance for pyridinic- $\mathrm{N}$ moieties in an acidic electrolyte can be ascribed to their protonation under such conditions. The additional electron that may donate the N-6 moieties and the redox processes should be impeded in this medium, producing a decrease in capacitance (Fig. 17). ${ }^{115}$ Furthermore, many previous reports have demonstrated that nitrogen functional groups played a positive role in electrochemical capacitors in organic electrolytes. ${ }^{90,116}$ Diego Cazorla-Amorós et al. observed an electrochemical response of $\mathrm{N}$-doped activated carbon fiber electrodes in a typical organic electrolyte (TEMA- $\mathrm{BF}_{4} / \mathrm{PC}$ ). The results demonstrated that the presence of aromatic nitrogen groups, especially positively charged $\mathrm{N}$-functional groups, greatly improved the stabilization of the capacitor due to an increase in the electrical conductivity of the electrode, increased stability of the material and a decrease in
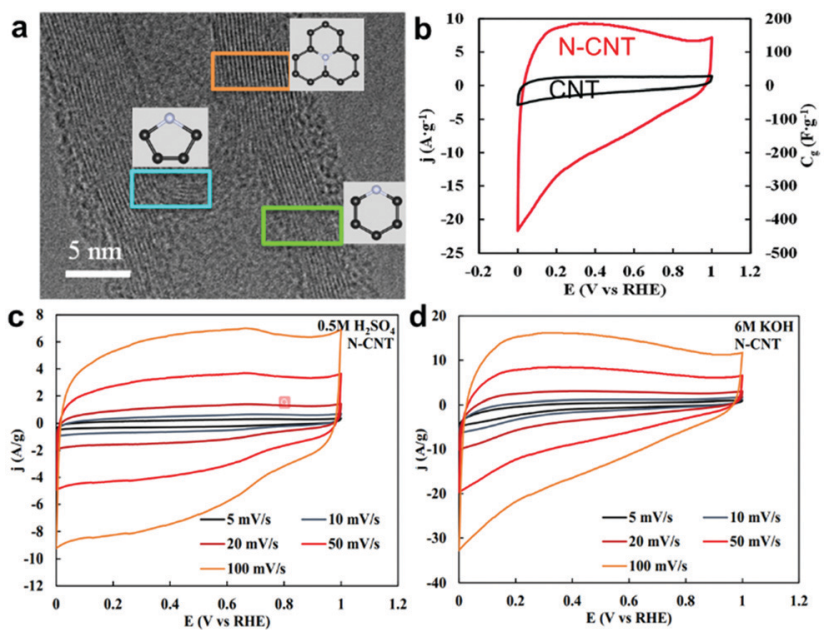

Fig. 17 (a) TEM images of the N-CNTs and three kinds of nitrogen configurations. (b) Cyclic voltammograms of N-CNT (red line) and CNT (black line) electrodes in $6 \mathrm{M} \mathrm{KOH}$ solutions at $50 \mathrm{mV} \mathrm{s}^{-1}$. Cyclic voltammetry of $\mathrm{N}-\mathrm{CNT}$ at different scan rates (c) in $0.5 \mathrm{M} \mathrm{H}_{2} \mathrm{SO}_{4}$ and (d) in $6 \mathrm{M} \mathrm{KOH}$. Reproduced with permission from ref. 115. Copyright 2014 The Royal Society of Chemistry.

electrolyte decomposition rate. ${ }^{117}$ In another example, Chi-Chang $\mathrm{Hu}$ et al. also showed that the introduction of nitrogen in the graphene skeleton endowed the nitrogen-doped reduced graphene oxide with enhanced conductivity, surface wettability and extra electrostatic interactions of organic ions. These properties were beneficial for enhancing the surface-charge-storage capacity. ${ }^{118}$ To sum up, $\mathrm{N}$ doping in carbon has shown potential application in supercapacitors, but the precise roles of the four most dominant nitrogen functionalities in different electrolytes are very ambiguous, which sometimes leads to contradictory results. Therefore, whether nitrogen doping is one of the central keys in the design of supercapacitor electrode materials needs further investigations.

\subsection{P-Doped porous carbons}

In comparison to nitrogen, phosphorus has a larger atomic radius and higher electron donating ability. Thus, $\mathrm{P}$ is a highly interesting candidate for altering the surface properties of carbon materials to meet the requirement of various applications. ${ }^{119-121}$ Generally, the presence of phosphorus in the carbon matrix is stable between 773 and $1273 \mathrm{~K}$. During the carbonization process at low temperatures, phosphorcarbonaceous species are created. Their content decreases by scission of the $\mathrm{P}-\mathrm{O}-\mathrm{C}$ bonds with an increase in the temperature, due to the growth of aromatic structures. ${ }^{122-124}$ In 2009, Hulicova-Jurcakova et al. first successfully prepared phosphorusrich microporous carbons by the phosphoric acid treatment of three carbon precursors, namely, fruit stones showing a capacitance of $192 \mathrm{~F} \mathrm{~g}^{-1}$, styrene-divinylbenzene copolymer showing a capacitance of $212 \mathrm{~F} \mathrm{~g}^{-1}$ and bismelimido-diphenylmethanedivinylbenzene copolymer showing a capacitance of $220 \mathrm{~F} \mathrm{~g}^{-1}$. Significantly higher capacitance values are obtained from P-carbons than from P-free carbons. Noteworthily, the introduction 

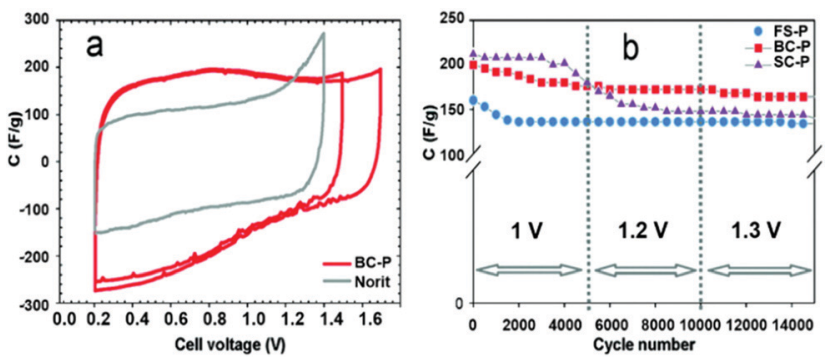

Fig. 18 (a) Cyclic voltammograms of $\mathrm{BC}-\mathrm{P}$ recorded at $5 \mathrm{mV} \mathrm{s}^{-1}$ and the cell voltage of 1.3 and $1.5 \mathrm{~V}$ compared with Norit at $1.2 \mathrm{~V}$. (b) Galvanostatic cycling at the current load of $5 \mathrm{~A} \mathrm{~g}^{-1}$ and cell voltage of $1,1.2$, and $1.3 \mathrm{~V}$. Reproduced with permission from ref. 125. Copyright 2009 American Chemical Society

of phosphorus endowed the resultant samples with stable operation at $1.5 \mathrm{~V}$ in aqueous electrolytes (Fig. 18)..$^{125}$ Jianan Yi et al. synthesized P-doped carbon with a hierarchical porous structure by the pyrolysis of lignocellulose immersed in $\mathrm{ZnCl}_{2} /$ $\mathrm{NaH}_{2} \mathrm{PO}_{4}$ aqueous solution. The higher intensity ratio $I_{\mathrm{D}} / I_{\mathrm{G}}$ of PCC than that of CC indicated that the P doping can effectively increase the defect quantity of biomass carbon. The as-obtained $\mathrm{P}$ doped porous carbon delivered a significant capacitance of $146 \mathrm{mF} \mathrm{cm} \mathrm{cm}^{-1}$ at a high current density of $10 \mathrm{~A} \mathrm{~g}^{-1}$ and outstanding rate performance. Furthermore, the P doped carbon electrode yielded a long-term cycling durability with more than $97.9 \%$ capacitance retention over 10000 cycles. ${ }^{126} \mathrm{Yu}$ et al. further incorporated phosphorus into hierarchical structured graphene, which was further used as an electrode and demonstrated outstanding surface redox behavior. As probed by in situ and ex situ spectroscopic techniques and density functional theory, the $\mathrm{P}=\mathrm{O}$ site of $\mathrm{C}-\mathrm{P}=\mathrm{O}$ bonding with the most favorable proton binding energy was identified and associated with highly reversible and fast pseudocapacitive behaviors. Benefiting from the synergistic effects of compositional and structural features, the P-incorporated graphene architectures showed improved specific capacitance, rate capability and cyclic stability in $1 \mathrm{M} \mathrm{H}_{2} \mathrm{SO}_{4}$ aqueous solution. ${ }^{127}$ Recently, the phosphorus-doped coal-based mesoporous carbon prepared by our team has been successfully applied as a supercapacitor electrode in organic electrolytes, which was capable of stable operation at voltages up to $3.0 \mathrm{~V}$, resulting in an enhanced energy density as high as $38.65 \mathrm{~W} \mathrm{~h} \mathrm{~kg}^{-1}$ at a current density of $1 \mathrm{~A} \mathrm{~g} \mathrm{~g}^{-1} \cdot{ }^{128}$ In another example, two-dimensional graphene was used as a simplified model for all $\mathrm{sp}^{2}$ hybrid carbon allotropes to explore the surface chemistry of P-doped carbon materials in $\mathrm{KOH}$ aqueous solution. It was identified that the $\mathrm{C} 3-\mathrm{P}=\mathrm{O}$ configuration was the most stable structure in the graphene lattice and played a key role in stabilizing the electrochemical interface between the electrode and electrolyte. To further disclose the advantages of P-doped graphene, the authors utilized density functional theory (DFT) calculations to understand the role of individual $\mathrm{P}$ atoms in various bonding configurations. The results illustrated that the strength of binding energy between $\mathrm{C}, \mathrm{P}$ and $\mathrm{O}$ near the Fermi level had a strong dependence on the number of $\mathrm{C}$ atoms connected to the $\mathrm{P}$ atom. Furthermore, the local density of states (DOS) of $\mathrm{C} 3-\mathrm{P}=\mathrm{O}$ was
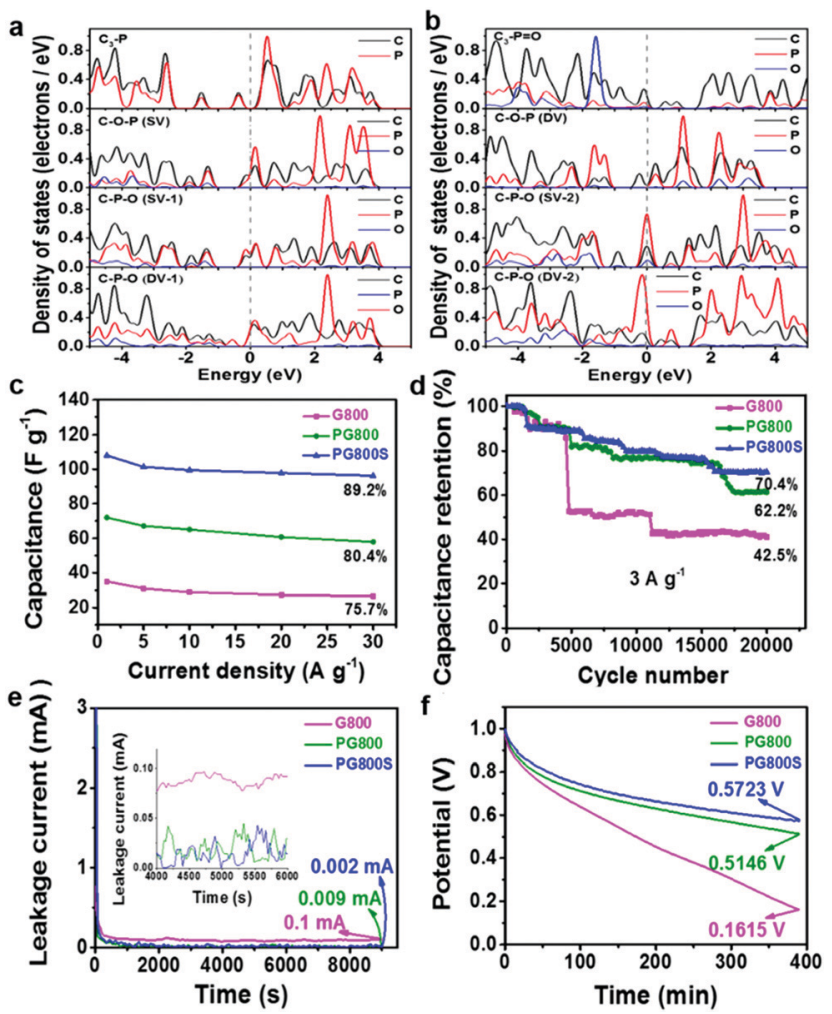

Fig. 19 ( $a$ and $b$ ) PDOS of different phosphorus configurations. The Fermi level is set to zero at the dotted line. (c) Variations in the gravimetric capacitance for a series of current densities. (d) Cycling stability of all three obtained samples at $3 \mathrm{~A} \mathrm{~g}^{-1}$. (e) Leakage current curves of all three obtained samples. (f) Self-discharging curves showing the open-circuit voltage changes over time for all three obtained samples. Reproduced with permission from ref. 64. Copyright 2019 American Chemical Society.

significantly stronger than that of the other configurations below the Fermi level, revealing that $\mathrm{C} 3-\mathrm{P}=\mathrm{O}$ was the most stable configuration. These results were consistent with the experimental results. These features enabled an electrode based on P-doped graphene to exhibit an enlarged potential window of $1.5 \mathrm{~V}$ in an aqueous electrolyte, a remarkable improved cycling stability and an ultralow leak current, as shown in Fig. $19 .{ }^{64}$ To conclude, for application in supercapacitors, phosphorusdoped carbon materials are very attractive because P-doping can increase defect sites, enlarge interlayer distance, facilitate the diffusion of both electrons and ions, and hence improve the electrochemical performance. More importantly, P-carbons play an obvious role in stabilizing the electrochemical interface between the electrode and electrolyte.

\subsection{B-Doped porous carbons}

Boron is another highly desired dopant in carbon materials. Boron enters the carbon lattice by substituting for carbon at the trigonal sites and serves as an electron acceptor owing to its three valence electrons, which can cause a shift in the Fermi level to the conducting band and hence tune the electronic structure of boron-doped carbon. ${ }^{69,129}$ Moreover, B atom in the carbon materials can facilitate charge transfer between 
neighboring carbon atoms and thus enhance their electrochemical performances. ${ }^{130,131}$ Therefore, the B-doped carbon material is a highly promising candidate for supercapacitor carbon electrodes. As in previous reports, boron doping can be accomplished through a diverse range of synthesis techniques such as laser ablation, ${ }^{132}$ arc discharge method, ${ }^{133}$ hydrothermal reaction, ${ }^{134}$ nano-casting approach, ${ }^{135}$ or chemical vapor deposition technique. ${ }^{136,137}$ Gao and co-workers prepared B-doped mesoporous carbon by the nano-casting approach. X-ray photoelectron spectroscopy (XPS) measurements indicated that the B content in carbon was as high as 2.9 at $\%$. When measured as a supercapacitor electrode, it showed a higher specific capacitance of $268 \mathrm{~F} \mathrm{~g}^{-1}$ than that of an undoped carbon $\left(221 \mathrm{~F} \mathrm{~g}^{-1}\right)$ at $5 \mathrm{mV} \mathrm{s}^{-1}$. The low solution resistance RS of $1.05 \mathrm{U}$ was also obtained due to the improved wettability after the incorporation of boron functional groups. ${ }^{135}$ Another study also indicated that minimal boron doping in amounts of 0.2 at\% was capable of boosting the specific capacitance by 1.6 times higher than pristine carbon. ${ }^{68}$ Besides, boron doped graphene oxide was prepared via a simple thermal annealing of $\mathrm{GO} / \mathrm{B}_{2} \mathrm{O}_{3}$ as shown in Fig. 20. The enhanced specific capacitance of $448 \mathrm{~F} \mathrm{~g} \mathrm{~g}^{-1}$ was achieved after boron doping without adding any conductivity enhancer, which resulted from the improved conductivity of the active material by boron doping. ${ }^{138}$ However, many previous works are mainly focused on adjusting the total boron content but not on controlling the boron configuration. Recently, M. Enterríet et al.
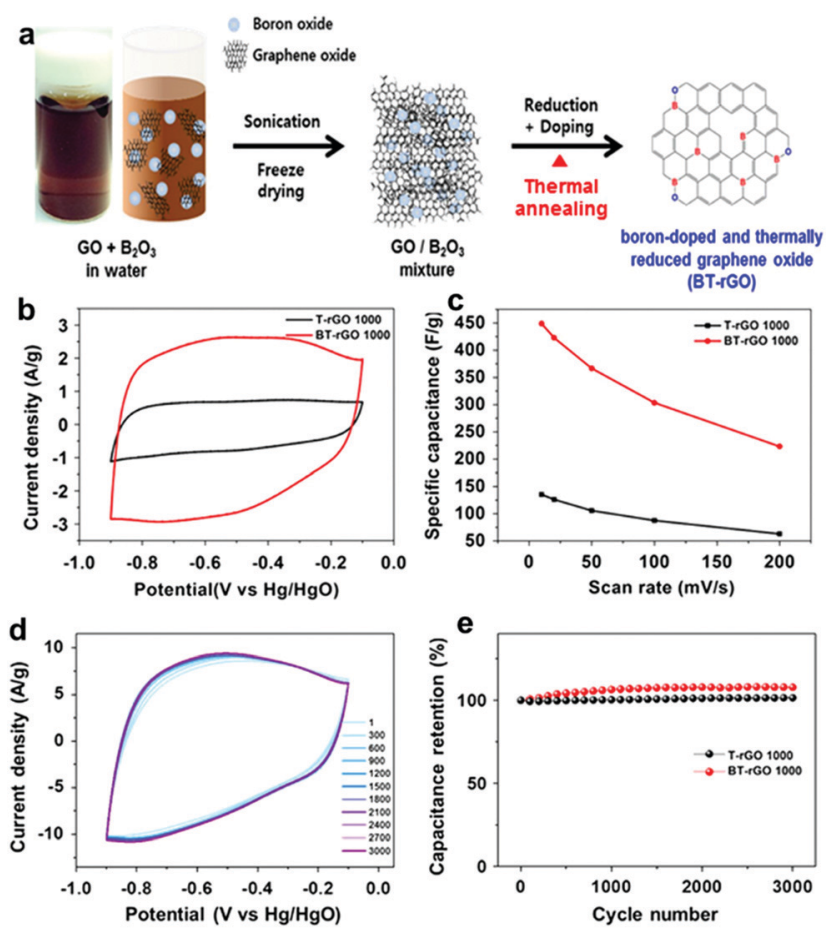

Fig. 20 (a) Schematic illustration of the preparation of BT-rGO. (b) CV curves at a scan rate of $10 \mathrm{mV} \mathrm{s}^{-1}$. (c) Specific capacitances as a function of scan rate. (d) CV curves for 3000 cycles at a scan rate of $50 \mathrm{mV} \mathrm{s}^{-1}$. (e) Capacitance retentions as a function of cycle number of T-rGO and BT-rGO electrodes. Reproduced with permission from ref. 138. Copyright 2015 Springer Nature. demonstrated that different configurations of boron doping have different contributions to the electrochemical performance. Both $\mathrm{BC}_{3}$ and $\mathrm{BC}_{2} \mathrm{O}$ configurations were derived from boron substituting atoms, which increased the electrical conductivity of the B-doping carbon electrode, while boron oxides including $\mathrm{BCO}_{2}$ and $\mathrm{B}_{2} \mathrm{O}_{3}$ induced interfacial redox reactions on the carbon surface in $1 \mathrm{M} \mathrm{H}_{2} \mathrm{SO}_{4}$ electrolyte. ${ }^{131}$ After that, Rosalynn Nankya et al. synthesized boron-doped mesoporous graphene by a hydrothermal process. They further varied the boron configuration in mesoporous graphene by annealing at high temperature and investigated the impact of different boron configurations on electrochemical performance in $6 \mathrm{M} \mathrm{KOH}$ electrolyte. As a result, the $\mathrm{BC}_{2} \mathrm{O}$ configuration of boron-doped mesoporous graphene showed enhanced kinetic performance due to the low diffusion resistance of electrolytes and high electrical conductivity. Yet, the $\mathrm{BCO}_{2}$ configuration in borondoped mesoporous graphene caused a faradaic redox reaction, resulting in high specific capacitance. ${ }^{139}$ Moreover, M. Sathish et al. have fabricated boron-doped graphene by supercritical fluid processing. They demonstrated that the prepared borondoped graphene electrode achieved a higher energy density of $39.3 \mathrm{~W} \mathrm{~h} \mathrm{~kg}^{-1}$ in $\mathrm{EMIMBF}_{4}$ and $18.6 \mathrm{~W} \mathrm{~h} \mathrm{~kg}^{-1} \mathrm{M} \mathrm{TEMA-BF}_{4} / \mathrm{PC}$ than that of bare graphene. ${ }^{140}$ The above works prove that mere doping with boron can pave the way towards the design of novel supercapacitor electrodes.

As aforementioned, various doping candidates including oxygen, nitrogen, phosphorus and boron have been widely introduced into the carbon matrix to improve the electrochemical performance of carbon-based supercapacitors. However, there is still an enormous space to optimize the supercapacitor performance. From recent reports, multi-heteroatom doping in carbon materials is found to be more effective in comparison with mono-heteroatom doping, since the co-doped carbon materials can exhibit a range of unique properties. Incorporation of dual- or triple-doping heteroatoms in the carbon materials provides unique chemical and electronic properties due to the creation of defects and uneven charge distribution in the carbon framework structure, which can further optimize the nano/microstructure and enhance the capacitive properties of carbon materials. Among frequently-used heteroatoms, $\mathrm{N}$ is one of the most popular dopants in carbon modification, while $\mathrm{O}, \mathrm{P}$, and $\mathrm{B}$ can serve as co-dopants in co-doped carbon framework structures.

\subsection{Dual-heteroatom doped porous carbons}

Relatively, $\mathrm{N}$ and $\mathrm{O}$ are easy to introduce into the carbon skeleton by direct carbonization of N,O-containing precursors and can modulate the hydrophilic properties and wettability of materials, contributing to pseudocapacitance. ${ }^{141-144}$ Hui Zhu and co-workers designed a N/O-co-doped activated carbon by an integrated one-step process directly from organic monomers. The N/O-dual doping in carbon can enhance both the electrical conductivity and surface hydrophilicity. Moreover, the large specific surface area of N/O-co-doped activated carbon was favourable for static charge storage and faradaic accumulation. ${ }^{141}$ As a result, the obtained sample exhibited superior capacitive behaviors such as large capacitance, high energy and 
power density, and long cycle life. Kehan Liang and co-workers successfully prepared the $\mathrm{N}, \mathrm{O}$ co-doped ordered mesoporous carbon (N/O-OMC) by the solid-solid grinding method using KIT-6 as a template, $\mathrm{Fe}\left(\mathrm{NO}_{3}\right)_{3}$ as a catalyst, and polyvinylpyrrolidone as a source of $\mathrm{C}, \mathrm{N}$, and $\mathrm{O}$. The N/O-OMCs exhibited double mesoporous structures, suitable $\mathrm{N}$ and $\mathrm{O}$ content and large specific surface area, which is kinetically favourable for the transportation of ions and electrons. ${ }^{145}$ Hulicova-Jurcakova and co-workers further studied the synergistic effect of nitrogen- and oxygen-containing surface functional groups of microporous activated carbon on its electrochemical performances in $1 \mathrm{M} \mathrm{H}_{2} \mathrm{SO}_{4}$, which was very important for the development of a high-performance carbon electrode for supercapacitors. The results revealed that pseudocapacitance originated from both the oxygen and the nitrogen containing functional groups located in pores larger than $10 \AA$. On the other hand, pores between $5 \AA$ and $6 \AA$ were most effective in double-layer formation, which correlated well with the size of hydrated ions. Although the quaternary and pyridinic- $N$-oxide nitrogen groups had enhanced effects on capacitance due to the positive charge, and thus an improved electron transfer at high current loads, the most important functional groups affecting energy storage performance were pyrrolic and pyridinic nitrogen along with quinone oxygen. ${ }^{146}$ Recently, Ziyang Song and co-workers proposed a generalized and robust route based on electroactive benzoquinone and amines with different chemical structures and constituents to design $\mathrm{O} / \mathrm{N}$ co-doped porous carbons (Fig. 21). This approach drives the designability of high-surface-area carbons with tailored geometries (nanosphere, nanofiber, granule and honeycomb), ensuring fast ion/electron transport kinetics to support electrical double layer capacitance. The highly redox-active $\mathrm{O} / \mathrm{N}$ heteroatoms triggered remarkable pseudocapacitance via reversible faradaic reactions of benzoquinone/hydroquinone transformation and pyridinic/pyrrolic nitrogen responses in a $\mathrm{H}_{2} \mathrm{SO}_{4}$ electrolyte. They also pointed out that the enhanced wetting compatibility of carbon surfaces/ electrolyte ions resulting from $\mathrm{O} / \mathrm{N}$ doping can further extend the carbon electrodes to various electrolyte systems. ${ }^{147}$ Furthermore, a number of N/O co-doped carbon materials have been developed for supercapacitors, such as the interconnected hierarchical porous carbon, three-dimensional (3D) interconnected architecture carbon sponge and so on. ${ }^{144,148,149}$

Besides, many groups have started focusing on co-doped carbon materials that not only have nitrogen, but also simultaneously phosphorus incorporated into their structures. A pioneering work in this context was reported by Woo and co-workers in 2012, who fabricated N/P-codoped carbon by pyrolysing mixtures of dicyandiamide, phosphoric acid and metal salts and subsequent elimination of metal residues using aqua regia. They proved that the P-doping enhanced the charge delocalization of the carbon atoms and constructed a morphology with many open edge sites that are split and wrinkled. As shown in Fig. 22, the additional P-doping altered the morphology of the NDC-based catalysts, resulting in the increased ORR activity of the carbons in comparison to merely N-doped materials. ${ }^{150}$ Then, many published studies proved

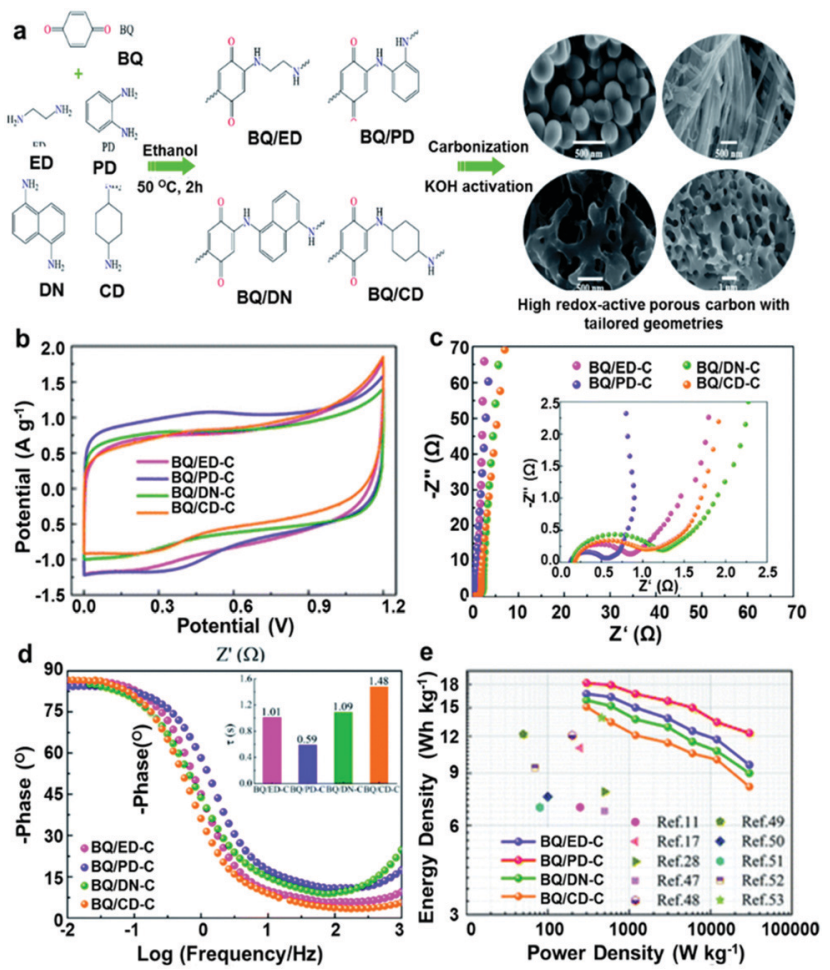

Fig. 21 (a) Chemical structures of the precursors and schematic synthetic route to $B Q / X-C$. Electrochemical evaluation of the symmetric supercapacitors based on BQ/X-C based devices: (b) CV curves, (c) Nyquist plots, (d) Bode plots and (e) Ragone plots. Reproduced with permission from ref. 147. Copyright 2020 The Royal Society of Chemistry.
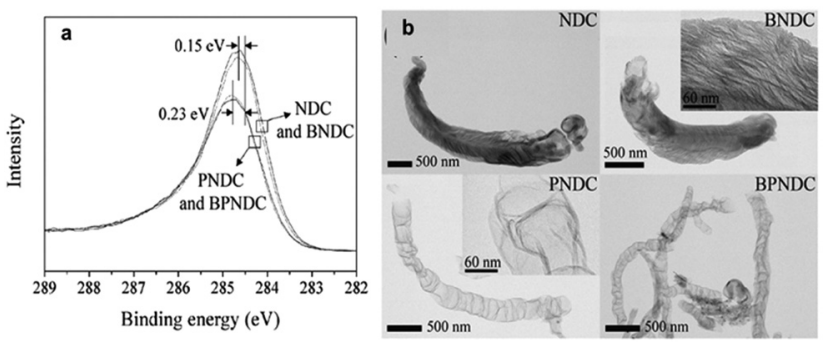

Fig. 22 (a) XPS-C1s spectroscopy and (b) TEM images of the prepared catalysts. Magnified images of BNDC and PNDC are inset in each figure. Reproduced with permission from ref. 150. Copyright 2012 American Chemical Society.

the potential of N/P-codoped carbon as supercapacitor electrodes. For example, Xiaodong Yan and co-workers fabricated phosphorus and nitrogen enriched porous carbons ( $\mathrm{P} / \mathrm{N}-\mathrm{PC})$ via direct heat treatments of $3 \mathrm{D}$ honeycomb-patterned $\mathrm{H}_{3} \mathrm{PO}_{4} / \mathrm{PAN}$ composite precursors. The surface N/P concentration and chemical structure can be well controlled by changing the carbonization temperature. Experiment results confirmed that such P/N-PC was an excellent electrode material for supercapacitors. ${ }^{151}$ Recently, Jutao Jin and co-workers reported porous carbon nanosheets doped with abundant nitrogen $(\mathrm{N})$ dopants in the basal plane and phosphorus (P) functional groups at the edge of the graphene lattice. The authors intensified their study by directly 
performing comparative measurements of purely N-doped and $\mathrm{P} / \mathrm{N}$ co-doped carbons. They pointed out that the crucial change came along with changes in the morphology. The SEM image of phosphorus and nitrogen co-doped porous exfoliated carbon nanosheets prepared at $950{ }^{\circ} \mathrm{C}$ (PN-ECB-950) showed an intertwined scaffold interconnected by wrinkle-rich thin graphite layers, while the $\mathrm{N}$-doped carbon sheet synthesized at $950{ }^{\circ} \mathrm{C}$ (N-ECB-950) without adding phosphoric acid showed an irregular morphology. The open porous framework and abundant nitrogen/phosphorus functional groups, combined with its large specific surface area, made the N-ECB-950 sample a highperformance active material for supercapacitor electrodes. ${ }^{152}$ More recently, Yan Ma and co-workers reported nitrogen and phosphorus co-doped carbon aerogels prepared by carbonization of the synthesized organic xerogel and subsequent activation by KOH. The optimized N/P co-doped carbon aerogel exhibited a high specific capacitance of $302 \mathrm{~F} \mathrm{~g}^{-1}$ at $0.5 \mathrm{~A} \mathrm{~g}^{-1}$ and good rate capability with a capacitance of $214 \mathrm{~F} \mathrm{~g}^{-1}$ retained at $20 \mathrm{~A} \mathrm{~g}^{-1}$, as well as excellent cycling stability with $100 \%$ retention after 20000 cycles in $6 \mathrm{M} \mathrm{KOH}$ electrolyte. The superior capacitance performance of the N/P co-doped carbon aerogel can be ascribed to the synergetic effect of $\mathrm{N}$ and $\mathrm{P}$ groups and the hierarchical porous structure. ${ }^{153}$ Theoretical calculations further disclosed that the dual doping of $\mathrm{N}$ and $\mathrm{P}$ could offer abundant electroactive sites, optimizing the charge distribution and co-activating the adjacent carbon atoms. ${ }^{154}$ Doping N/P dual atoms into hierarchical porous carbons has also been achieved by different approaches. ${ }^{155-157}$ Furthermore, an interesting finding was that under the synergistic effect of $\mathrm{P}$ and $\mathrm{N}$, starch has been successfully transformed from natural organic macromolecule compounds into carbon materials without morphology collapse (Fig. 23a), which was demonstrated by Cheng-Meng Chen' group. Further, in situ Fourier transform infrared and in situ nuclear magnetic resonance combined with the in situ X-ray diffraction technique have been used to investigate the whole evolution process from natural organic macromolecule compounds to carbon materials. It has been disclosed that $\mathrm{N}$ atoms contributed to the cyclization and growth of polyaromatic units, while $\mathrm{P}$ was responsible for the formation of phosphate and polyphoshate bridges that cross-link polyaromatic unit fragments into graphitic crystallines (Fig. 23b). ${ }^{158}$ This resulted in the high carbon yield and the inherited natural spherical morphology, which is highly desired for the design of the advanced carbon electrode. N/B co-doped carbon material, as one of the multi-heteroatoms co-doping carbon materials, becomes more and more concerned. ${ }^{159}$ This can be ascribed to the following merits. On the one hand, the atomic radius of $\mathrm{B}$ and $\mathrm{N}$ is similar to that of C, thus the N/B co-doped carbon can keep the planar structure of carbon without obvious structure deformation. ${ }^{160}$ On the other hand, a B atom can be used as an electron acceptor owing to its three valence electrons, leading to a Fermi level shift to the conducting band and thus regulating the electronic structure of the carbon material. ${ }^{66,69}$ Furthermore, N-doping can enhance the electrolyte ion adsorption energy due to the higher electronegativity of $\mathrm{N}$ (3.04) than that of $\mathrm{C}(2.55) .{ }^{161}$ Benefiting from the synergistic coupling effects and well-maintained $\mathrm{sp}^{2}$

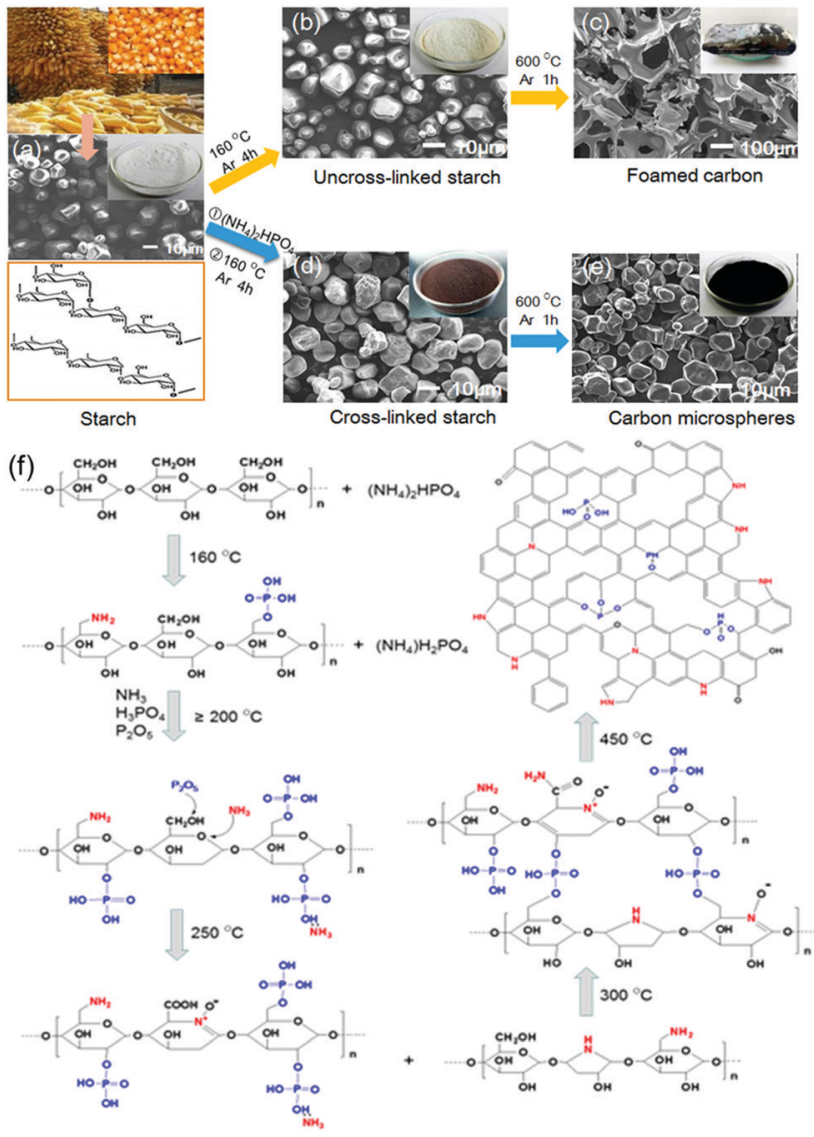

Fig. 23 The preparation process of foamed carbon and spherical carbon and the structural evolution at the different stages $(a-e)$. Possible scheme of chemical evolution during thermal treatment for the cross-linking spherical carbon (f). Reproduced with permission from ref. 158. Copyright 2019 American Chemical Society.

hybridized graphitic configuration, various $\mathrm{B} / \mathrm{N}$ co-doping carbon materials have been fabricated to regulate the pristine band structure and surface states so as to enhance the electrochemical performances of supercapacitors. ${ }^{162-164}$ For example, Dong Guo et al. synthesized a B/N co-doped porous carbon foam by pyrolyzing a mixture of starch with urea and boric acid. Electrochemical measurements performed in direct comparison to N-doped, B-doped and pristine derivatives of the material have revealed that significant synergies can be achieved by dual doping. Benefiting from the high-level N (9.38 at\%) and B (3.87 at\%) co-doping and synergistic effects, the obtained BNCNFs showed a superior specific capacity of $402 \mathrm{~F} \mathrm{~g}^{-1}$ at $0.5 \mathrm{~A} \mathrm{~g}^{-1}$, and the specific capacity remains at $266 \mathrm{~F} \mathrm{~g}^{-1}$ even at $20 \mathrm{~A} \mathrm{~g}^{-1}$ in $6 \mathrm{M} \mathrm{KOH}^{165}$ To improve the doping efficiency, Hao Chen and co-workers reported a facile solvent evaporation induced self-assembly method in combination with an inexpensive $\mathrm{SiO}_{2}$ hard template to prepare $\mathrm{B} / \mathrm{N}$ co-doped hierarchical porous carbon (BN-HPC). The obtained BN-HPC contained $3.97 \mathrm{wt} \% \mathrm{~B}$ and $12.10 \mathrm{wt} \% \mathrm{~N}$. The high content of boron and nitrogen not only afforded pseudocapacitance but also improved the wettability of carbons to the electrolyte. With these effects working together, BN-HPC exhibited excellent electrochemical 
performance in supercapacitors. Furthermore, the authors further suggested that a certain $\mathrm{C}-\mathrm{N}-\mathrm{B}$ configuration also caused an enhanced pseudocapacitance. ${ }^{66}$ The uniform doping configuration in the carbon framework became a key factor to achieve high-performance co-doped carbon electrode materials. Recently, Zhichao Zhao and Yibing Xie designed binder-free boron and nitrogen co-doped carbon (BNDC) nanowires by electro-polymerizing aniline using boric acid as a boron source and polyvinyl pyrrolidone as a structure-directing agent, and subsequent carbonization. The as-formed BNDC featured uniform distribution and appropriate contents of boron and nitrogen in the carbon skeleton, a porous structure with rational surface area as well as an interconnected network structure. When used as an electrode material for supercapacitors, the binder-free BNDC/CP electrode exhibited a high specific capacitance of $504.0 \mathrm{~F} \mathrm{~g}^{-1}$ at $1.0 \mathrm{~A} \mathrm{~g}^{-1}$ and cycling capacitance retention of $97.4 \%$ after 10000 cycles at $10.0 \mathrm{~A} \mathrm{~g}^{-1}$. Interestingly, the authors fabricated a symmetrical solid-state BNDC supercapacitor using the $\mathrm{BNDC} / \mathrm{CP}$ electrode and $\mathrm{PVA}-\mathrm{H}_{2} \mathrm{SO}_{4}$ gel electrolyte. As expected, the BNDC supercapacitor showed not only remarkable specific capacitance of $255.7 \mathrm{~F} \mathrm{~g}^{-1}$ at $1.0 \mathrm{~A} \mathrm{~g}^{-1}$ and high energy density of $22.7 \mathrm{~W} \mathrm{~h} \mathrm{~kg}{ }^{-1}$ at a power density of $0.2 \mathrm{~kW} \mathrm{~kg}^{-1}$, but also excellent cycling stability of $91.0 \%$ capacitance retention after 5000 cycles at $5.0 \mathrm{~A} \mathrm{~g}^{-1}$, as well as good flexibility. ${ }^{166}$ Recently, the ongoing process of $\mathrm{B} / \mathrm{N}$ co-doped carbon materials for supercapacitors has been presented in a study by Xiaoxiao Zou et al. The authors reported a facile yet efficient one-step doping approach to directly synthesize BNHGA using ammonia borane $\left(\mathrm{NH}_{3} \mathrm{BH}_{3}\right)$ as a triple functional reagent. As illustrated in Fig. 24, the as-prepared boron and nitrogen co-doped holey graphene aerogel (BN-HGA) possesses a hierarchical 3D network for fast ion diffusion and adsorption in the high-viscosity gel electrolyte. The integrity of the carbon matrix was favorable for high electronic conductivity. Furthermore, the rich $\mathrm{B}-\mathrm{N}$ motifs in the carbon matrix gave rise to localized surface polarization providing highly reversible redox sites for stable pseudo-capacitance. Finally, the good mechanical strength and flexibility of the as-prepared BN-HGA made it a promising electrode material for all-solid-state flexible supercapacitors. ${ }^{161}$ It can be concluded that $\mathrm{B} / \mathrm{N}$ co-doping is indeed a promising concept for the design and development of new carbon electrode materials for supercapacitors.

\subsection{Tri-heteroatom doped porous carbons}

Although tri-heteroatom doped carbon materials have been relatively less studied in comparison to mono- or dualheteroatom doped carbon materials, tri-heteroatom doping can also lead to in some cases better electrochemical performances. ${ }^{167-170}$ As above reviewed, $\mathrm{O}$ and $\mathrm{N}$ heteroatoms can effectively induce pseudocapacitance of the carbon surface due to electron-acceptor/electron-donor properties and regulate the hydrophilic properties of carbon. B-doping can modify the electronic structure of carbon, facilitate charge transfer and enhance the electrochemical performance. Owing to its low electronegativity and large atom size, P-doping can change the local charge density, along with creation of more structural

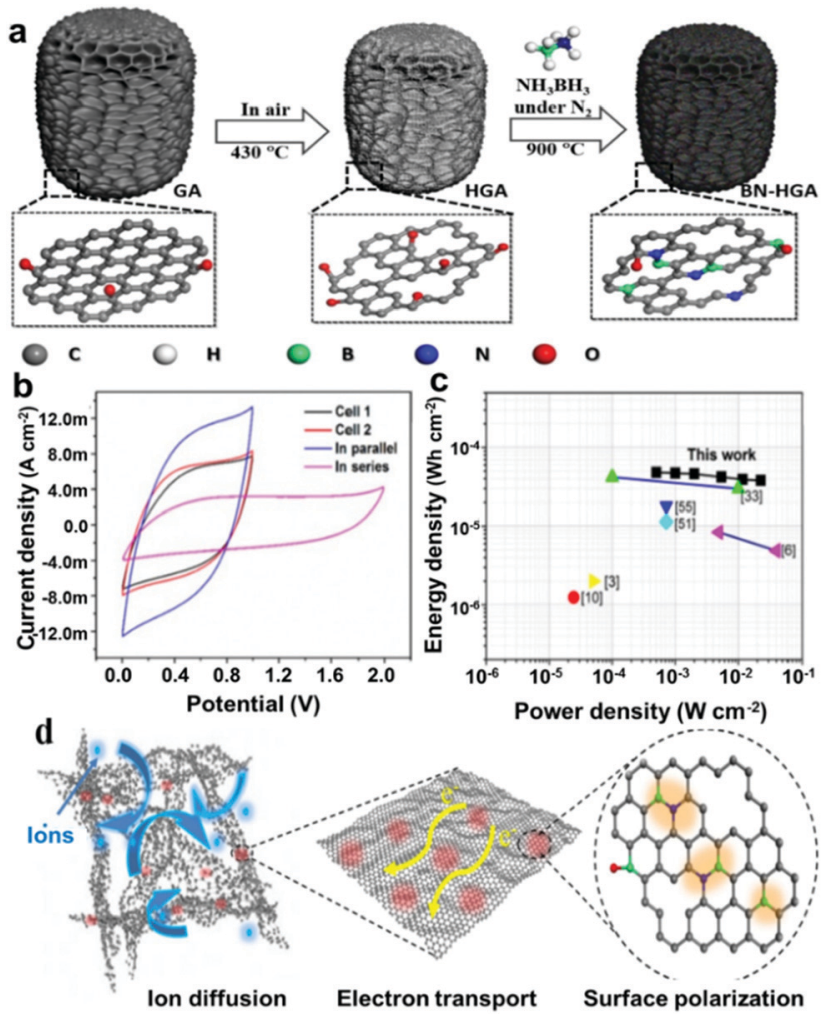

Fig. 24 (a) Schematic diagram on the preparation process of $B / N$ co-doped holey graphene aerogels. (b) CVs for two FSCs in series or in parallel connection. (c) Ragone plot for the FSCs with areal energy/power densities and comparisons with other literatures data. (d) Schematic illustration of capacitance performance of B/ $\mathrm{N}$ co-doped holey graphene aerogels. Reproduced with permission from ref. 161. Copyright 2019 Elsevier Ltd.

disorders, blocking the nonstable active oxidation sites and widening the potential window. Therefore, the full utilization of the synergistic effect of multiple-element doping in the carbon framework could bring a capacitive strategy to achieve excellent capacitive performances. Beneficial effects on the electrochemical performance of multiple-element doping in carbon electrodes have been observed by Huarong Peng and co-workers. They used a combination of a soft-template method and freeze-drying as well as chemical etching to fabricate a $\mathrm{N}, \mathrm{O}, \mathrm{S}-\mathrm{enriched}$ carbon foam (NOSC). The pore engineered carbon framework with outstanding specific surface area can allow efficient ion diffusion and maximize the effective interfacial area. Furthermore, the pore engineered carbon structure was also uniformly doped with $\mathrm{N}$, O, and $\mathrm{S}$, which facilitated charge transfer and thus improved the EDLC of carbon foam. By comparison, it was found that NOSC had a higher specific capacitance of $402.5 \mathrm{~F} \mathrm{~g}^{-1}$ at $1 \mathrm{~A} \mathrm{~g}^{-1}$ and a superior rate capability of $308.5 \mathrm{~F} \mathrm{~g}^{-1}$ at $100 \mathrm{~A} \mathrm{~g}^{-1}$ than that of the NOC. This work exemplified how to integrate multifunctional heteroatoms with a rationally designed porous structure to improve the capacitance performance of carbon structures. ${ }^{171}$ Recently, the direct incorporation of heavier dopants into the carbon framework has also been regarded as an efficient way 


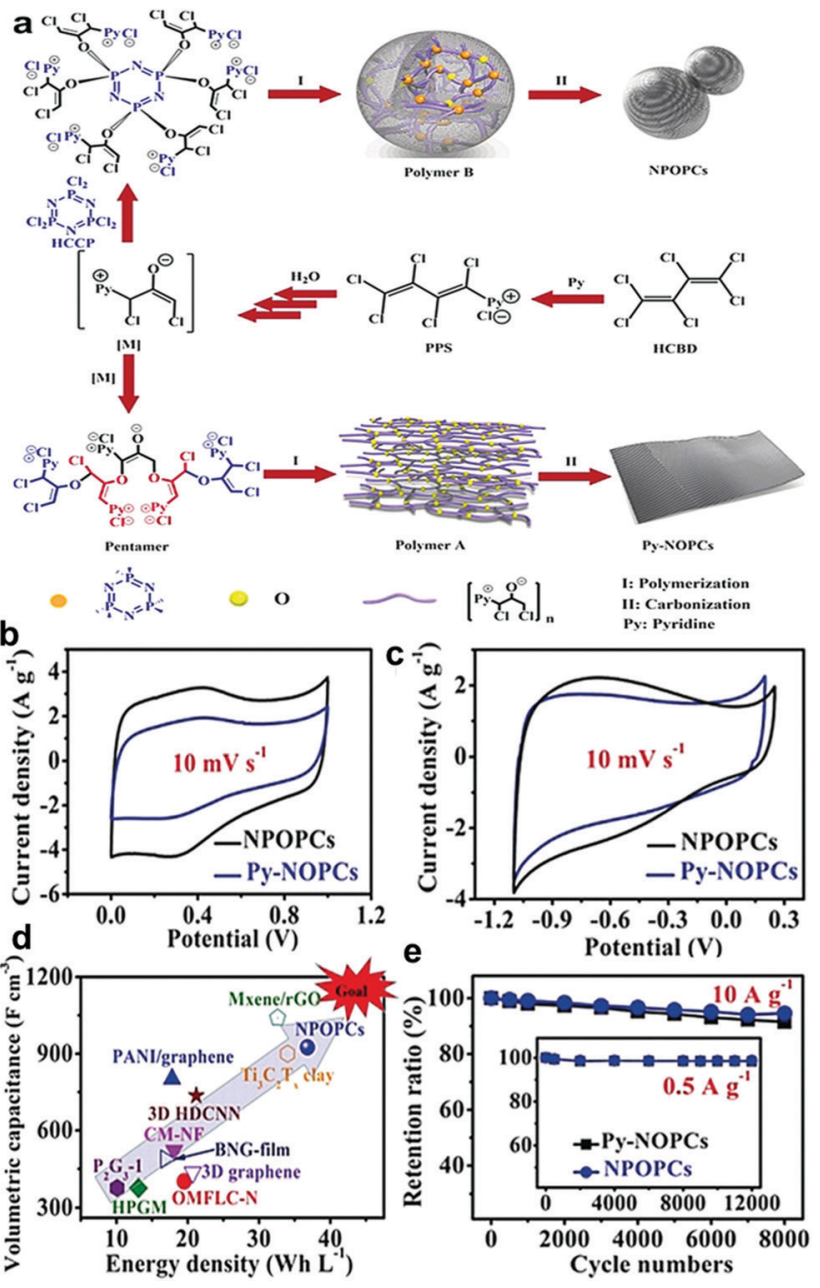

Fig. 25 (a) Proposed polymerization and carbonization route of Py-NOPCs and NPOPCs. Electrochemical evaluation of Py-NOPCs and NPOPCs: cyclic voltammetry (CV) tests at $10 \mathrm{mV} \mathrm{s}^{-1}$ in the first cycle, in $0.5 \mathrm{~m} \mathrm{H}_{2} \mathrm{SO}_{4}$ (Py-NOPCs) (b) and $6 \mathrm{~m} \mathrm{KOH}$ (NPOPCs) (c). (d) Volumetric specific capacitance vs. energy density for different materials. (e) Cycling performance of NPOPCs in $6 \mathrm{~m} \mathrm{KOH}$ at a current density of $10 \mathrm{Ag}^{-1}$ (inset: cycling performance at a current density of $0.5 \mathrm{Ag}^{-1}$ ). Reproduced with permission from ref. 172. Copyright 2019 Wiley-VCH Verlag GmbH \& Co. KGaA, Weinheim.

for enhanced natural density as well as better volumetric capacitance. Huile Jin and co-workers reported a new class of high-density heteroatom-doped porous carbons that can be used as an aqueous-based supercapacitor material. The synthesis of heteroatom-doped carbon is shown in Fig. 25a. The obtained N-, P- and O-doped porous carbon spheres (NPOPCs) were highly doped by various heteroatoms, leading to a high packing density of $2.13 \mathrm{~g} \mathrm{~cm}^{-3}$ and abundant multimodal pores. The NPOPC-based symmetric supercapacitor cell exhibited an impressive volumetric specific capacitance of $760 \mathrm{~F} \mathrm{~cm}^{-3}$ at $1 \mathrm{~A} \mathrm{~g}^{-1}$ in $6 \mathrm{M} \mathrm{KOH}$ and an exceptional volumetric energy density of $36.8 \mathrm{~W} \mathrm{~h} \mathrm{~L}^{-1}$ (Fig. 25b-o). ${ }^{172}$ Yufeng Zhao and co-workers also produced $\mathrm{N}-\mathrm{P}-\mathrm{O}$ co-doped high density $3 \mathrm{D}$ graphene through a one-pot red phosphorus-assisted 'cutting-thin' technique. The as-prepared graphene exhibited a continuously 3D hierarchical porous structure with an average pore size of $6.1 \mathrm{~nm}$ and high density of $0.996 \mathrm{~g} \mathrm{~cm}^{-3}$. Additional computational studies have implied that the $\mathrm{N}-\mathrm{P}-\mathrm{O}$ co-doping can significantly enhance the charge delocalization with benefited electrochemical activity. Owing to the synergistic effect of multiple-element doping, the $3 \mathrm{D}$ hierarchical porous graphene (3D-HPG) as the supercapacitor electrode offered an ultrahigh specific capacitance of $426 \mathrm{~F} \mathrm{~g}^{-1}$ and volumetric specific capacitance of $424 \mathrm{~F} \mathrm{~cm}^{-3}$ at $1 \mathrm{~A} \mathrm{~g}^{-1}$ in $1 \mathrm{M} \mathrm{KOH}$ electrolyte. ${ }^{173}$ Actually, biomass materials possess an abundant porous structure and internally installed heteroatoms apart from their natural availability and high carbon content, so heteroatom-doped carbon materials with different dopant species and improved physical/ chemical properties for supercapacitors can easily be obtained from them. In fact, there have been many reports about biomass-derived heteroatom-doped carbon materials for supercapacitor application. ${ }^{169,174,175}$ Wei Zhou and co-workers employed a one-step pyrolysis carbonization of green bristlegrass seeds with the assistance of gelatinization to prepare $\mathrm{N} / \mathrm{S} / \mathrm{O}$ co-doped honeycomb-like porous carbon materials. Benefitting from their three-dimensional interconnected honeycomb-like hierarchical porous architecture together with high specific surface area and multi-heteroatom doping, the asobtained carbon materials exhibit a high specific capacitance of $391 \mathrm{~F} \mathrm{~g}^{-1}$ at $0.5 \mathrm{~A} \mathrm{~g}^{-1}$, good rate capability with $67.8 \%$ capacitance retention at $50 \mathrm{~A} \mathrm{~g}^{-1}$ in $6 \mathrm{M} \mathrm{KOH}$ electrolyte, and outstanding cycling stability with a capacitance retention of 97.2\% over 10000 cycles. ${ }^{175}$ Another work of N,O,S co-doped carbon materials has been performed by Liu Wan et al., who used one-step pyrolysis of chestnut shell and melamine to synthesize a N,O,S co-doped porous graphitic carbon. By tuning the carbonization temperature, the chestnut shell-based carbon prepared at $800{ }^{\circ} \mathrm{C}$ (CSC-800) exhibited a more graphitic N,O,S co-doped porous carbon with nitrogen content of up to 3.79 at\%, oxygen content of 13.35 at $\%$, and sulfur content of 0.52 at $\%$. Thanks to the optimized pore structure and rich surface heteroatoms, when applied as an electrode material in supercapacitors, the CSC-800 showed a high specific capacitance of $402.8 \mathrm{~F} \mathrm{~g}^{-1}$ at $0.5 \mathrm{~A} \mathrm{~g}^{-1}$ and excellent rate capability in $6 \mathrm{M} \mathrm{KOH}$ electrolyte. ${ }^{176}$ Peanut meal, a byproduct of the extraction of peanut oil, with $\mathrm{N}$ (7 at\%), P (2.49 at\%), and S (0.96 at\%) elements has directly been used as a precursor for nitrogen, phosphorus, and sulfur (S) self-doped hierarchically porous carbon (N-P-S-HPC), as shown in Fig. 26a. The water contact angle of PMC $\left(130^{\circ}\right), \mathrm{AC}\left(110^{\circ}\right)$, and N-P-S-HPC $\left(83^{\circ}\right)$ shown in Fig. 26b illustrated that N-P-S-HPC possessed the best wettability to the electrolyte, which was beneficial to the ion transfer. The obtained N-P-SHPC electrode exhibited an ultrahigh specific capacitance of $525 \mathrm{~F} \mathrm{~g}^{-1}$ at $1 \mathrm{~A} \mathrm{~g}^{-1}$ with the contributions of electrical double layer capacitance and pseudocapacitance, good rate capability with a capacitance retention of $68 \%$ at $10 \mathrm{~A} \mathrm{~g}^{-1}$ and excellent cycling stability (Fig. 26c and d). ${ }^{177}$ From these reports, it can be concluded that the intrinsic composition and natural structural features of various biomass materials have afforded them considerable potential as the precursors for multi-heteroatom co-doped carbon materials in supercapacitors. 

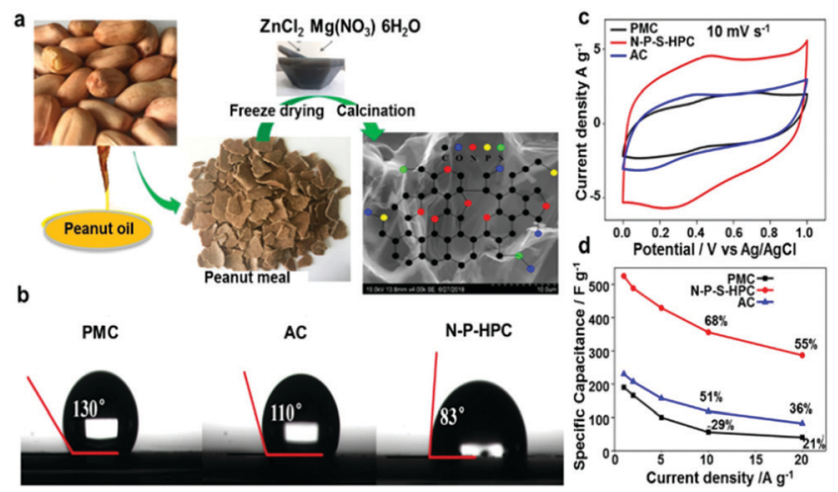

Fig. 26 (a) The preparation process of N-P-S-HPC. (b) Water contact angles of PMC, AC, and N-P-S-HPC. (c) CV curves of PMC, AC, and $\mathrm{N}-\mathrm{P}-\mathrm{S}-\mathrm{HPC}$ at $10 \mathrm{mV} \mathrm{s}^{-1}$ in $1 \mathrm{M} \mathrm{H}_{2} \mathrm{SO}_{4}$. (d) Specific capacitances of PMC, $\mathrm{AC}$, and $\mathrm{N}-\mathrm{P}-\mathrm{S}-\mathrm{HPC}$ from 1 to $20 \mathrm{~A} \mathrm{~g}^{-1}$. Reprinted with permission from ref. 177, Copyright 2019 American Chemical Society.

\section{Conclusions and prospects}

This review intended to offer the reader an overview of the most recent developments of various in situ experimental methods for porous carbon materials, the principles behind the experimental methods and the related data analysis methods. With the rapid development of in situ characterization (NMR, EQCM, IR, and scattering methods) and simulations (Fig. 27), it provides us with a powerful tool to probe a 'black box' of supercapacitor electrodes at the molecular scale, which can be a decisive step towards optimizing and improving the energy storage capabilities of carbon-based supercapacitors. Thus, with the aid of in situ characterizations, to control the charge storage mechanisms through appropriate combination of ions and solvents as well as electrode structures may be a useful strategy to tune the energy and power performances of

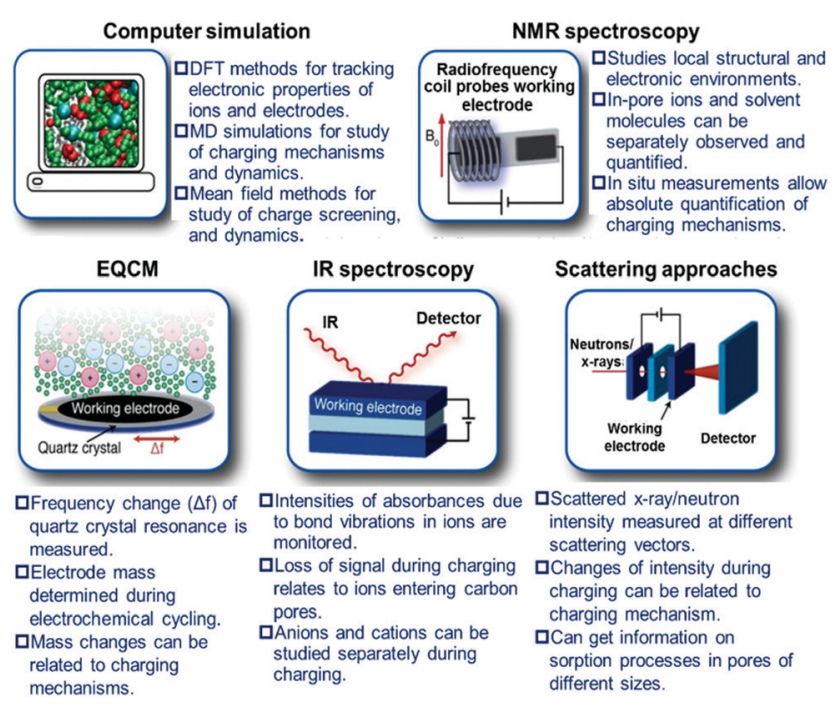

Fig. 27 Schematic showing the different approaches for studying the charging mechanisms of supercapacitors, and their advantages and challenges. Reproduced with permission from ref. 8 Copyright 2016 American Chemical Society. supercapacitors for a range of different applications. Furthermore, it is of no doubt that the practical application of a supercapacitor depends on the successful full cell configuration. The supercapacitor is mainly composed of the electrode and the separator as well as the electrolyte. Abundant carbon-based electrodes have been studied widely; however, the research on the two others could not be ignored. For example, the choice of the type and concentration of electrolyte has a great influence on the electrochemical behaviours of porous carbon electrodes. Finally, the current reliable in situ characterizations are also applicable to the in situ studies of various devices such as asymmetric supercapacitors, Li-ion batteries, Li-S batteries, Na-ion storage and fuel cells, etc.

Various heteroatom-doped carbons, including mono(O-, N-, P-, and B-doped carbons) or multi-doped porous carbon materials (dual-doped and triple-doped carbons), have been fabricated successfully via different methods and some promising results have been achieved over the past few years. As discussed in this article, the introduction of various heteroatoms within the carbon matrix can induce the following shared effects: (1) increased extrinsic defects; (2) high electronic mobility; and (3) enhanced wettability. Benefiting from these properties, the heteroatom doped porous carbon showed improved electrochemical performances. Furthermore, both $\mathrm{B}$ and $\mathrm{N}$ are relatively easier to enter the carbon lattice to tune the electronic structure of the doped carbon, while $\mathrm{B}$ and $\mathrm{N}$ play an obvious role in blocking the nonstable active oxidation sites and further stabilizing the electrochemical interface between the electrode and electrolyte. Particularly, the functional mechanisms of various heteroatom dopants with electrolyte ions are greatly different due to their different size and electronegativity. Therefore, according to the application, reasonable selection of dopants and accurate control of the doping configurations and their relative ratios, and compositions of co-dopants are highly desired. Besides, most of the studies in the literature use either aqueous alkaline or acidic electrolytes, which are not commercially feasible. Organic, gel and ionic liquid electrolytes to broaden the voltage window, as a key component in building and improving the overall supercapacitor performance, should be considered. For multi-doped carbon materials, especially for triple-doped carbons, the fundamental understanding of the synergetic promotion effect of different heteroatoms on electrochemical performances is still at its first stage of comparisons of apparent capacitive properties. Therefore, further research on how the multiple types of hetero-element dopant species work together in enhancing the electrochemical performances of these electrode materials is highly desirable. Furthermore, to fully understand the reaction mechanism of heteroatom-doped carbons during the charging/discharging process, in situ characterizations as well as advanced theoretical calculations may be required. The results, in turn, may promote the design and development of more efficient and ideal supercapacitor electrodes. Last but not the least, electrochemical properties of the supercapacitor electrode materials in the literature are usually focused on capacity, cycle stability, coulombic efficiency, energy density and power density, which are not 


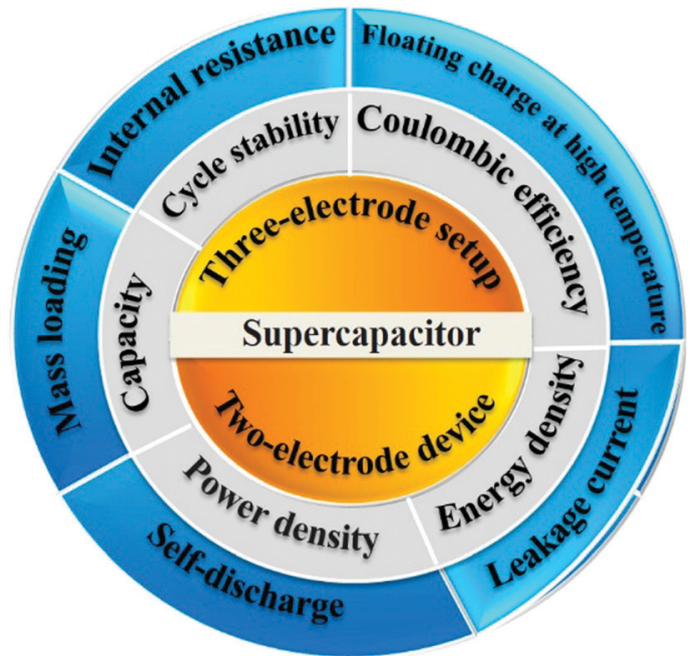

Fig. 28 Summary of necessary performance parameters to realize electrode materials accessible to practical applications.

aligned with their practical applications in an actual supercapacitor cell. To realize electrode materials accessible to practical applications, other performance parameters, such as the mass loading, internal resistance, self-discharge, leakage current as well as floating charge at higher temperature, ${ }^{178}$ should be assessed as shown in Fig. 28. Turning to improve performances under industrial requirements may be more meaningful for research itself.

\section{A statement of contributions}

\begin{tabular}{ll} 
Name of authors & Contributions \\
\hline $\begin{array}{l}\text { Chengmeng Chen } \\
\text { Lijing Xie }\end{array}$ & $\begin{array}{l}\text { Revision of the full-paper } \\
\text { Responsible for the abstract, } \\
\text { introduction and conclusion }\end{array}$ \\
Fangyuan Su, Zonglin Yi, & $\begin{array}{l}\text { Responsible for electrochemical } \\
\text { charge-storage mechanisms of } \\
\text { Zhenbing Wang }\end{array}$ \\
porous carbon for EDLCs and the \\
collection of the respective pictures \\
Riaoqian Guo & $\begin{array}{l}\text { Responsible for N-doped porous } \\
\text { carbons } \\
\text { Responsible for O-doped porous } \\
\text { carbons } \\
\text { Responsible for P-doped porous } \\
\text { carbons } \\
\text { Guohua Sun }\end{array}$ \\
Aziz Ahmad & $\begin{array}{l}\text { Responsible for B-doped porous } \\
\text { carbons } \\
\text { Responsible for dual-heteroatom } \\
\text { doped porous carbons } \\
\text { Responsible for tri-heteroatom doped } \\
\text { porous carbons and the collection of } \\
\text { pictures on heteroatom doped } \\
\text { porous carbons }\end{array}$ \\
&
\end{tabular}

\section{Conflicts of interest}

There are no conflicts to declare.

\section{Acknowledgements}

The authors gratefully acknowledge financial support from the National Science Foundation for Excellent Young Scholars of China (21922815), Key Research and Development (R\&D) Projects of Shanxi Province (201903D121180, 201903D121007), and the Scientific and Technological Key Project of Shanxi Province (20191102003).

\section{Notes and references}

1 F. Béguin, V. Presser, A. Balducci and E. Frackowiak, Carbons and Electrolytes for Advanced Supercapacitors, Adv. Mater., 2014, 26, 2219-2251.

2 J. W. Xin Li, One-dimensional and two-dimensional synergized nanostructures for high-performing energy storage and conversion, InfoMat, 2020, 2, 3-32.

3 A. Chen, X. Zhang and Z. Zhou, Machine learning: accelerating materials development for energy storage and conversion, InfoMat, 2020, 2, 553-576.

4 B. D. McCloskey, Expanding the Ragone Plot: Pushing the Limits of Energy Storage, J. Phys. Chem. Lett., 2018, 6, 3592-3593.

5 L. Liu, H. P. Zhao and Y. Lei, Advances on threedimensional electrodes for micro-supercapacitors: A minireview, InfoMat, 2019, 1, 74-84.

6 R. Wang, M. J. Yao and Z. Q. Niu, Smart supercapacitors from materials to devices, InfoMat, 2020, 2, 113-125.

7 A. C. Forse, J. M. Griffin, C. Merlet, J. Carretero-Gonzalez, A.-R. O. Raji, N. M. Trease and C. P. Grey, Direct observation of ion dynamics in supercapacitor electrodes using in situ diffusion NMR spectroscopy, Nat. Energy, 2017, 2, 16216.

8 A. C. Forse, C. Merlet, J. M. Griffin and C. P. Grey, New Perspectives on the Charging Mechanisms of Supercapacitors, J. Am. Chem. Soc., 2016, 138, 5731-5744.

9 C. Liu, X. Yan, F. Hu, G. Gao, G. Wu and X. Yang, Toward Superior Capacitive Energy Storage: Recent Advances in Pore Engineering for Dense Electrodes, Adv. Mater., 2018, 30, 1705713.

10 M. Sevilla and R. Mokaya, Energy storage applications of activated carbons: supercapacitors and hydrogen storage, Energy Environ. Sci., 2014, 7, 1250-1280.

11 A. C. Forse, J. M. Griffin, C. Merlet, P. M. Bayley, H. Wang, P. Simon and C. P. Grey, NMR Study of Ion Dynamics and Charge Storage in Ionic Liquid Supercapacitors, J. Am. Chem. Soc., 2015, 137, 7231-7242.

12 J. Yan, S. Li, B. Lan, Y. Wu and P. S. Lee, Rational Design of Nanostructured Electrode Materials toward Multifunctional Supercapacitors, Adv. Funct. Mater., 2019, 30, 1902564. 
13 J. Chmiola, G. Yushin, Y. Gogotsi, C. Portet, P. Simon and P. L. Taberna, Anomalous Increase in Carbon Capacitance at Pore Sizes Less Than 1 Nanometer, Science, 2006, 313, 1760-1763.

14 P. Simon and Y. Gogotsi, Materials for electrochemical capacitors, Nat. Mater., 2008, 7, 845-854.

15 Q. Gao, L. Demarconnay, E. Raymundo-Piñero and F. Béguin, Exploring the large voltage range of carbon/ carbon supercapacitors in aqueous lithium sulfate electrolyte, Energy Environ. Sci., 2012, 5, 9611-9617.

16 X. Wang, H. Zhou, E. Sheridan, J. C. Walmsley, D. Ren and D. Chen, Geometrically confined favourable ion packing for high gravimetric capacitance in carbon-ionic liquid supercapacitors, Energy Environ. Sci., 2016, 9, 232-239.

17 C. Merlet, B. Rotenberg, P. A. Madden, P.-L. Taberna, P. Simon, Y. Gogotsi and M. Salanne, On the molecular origin of supercapacitance in nanoporous carbon electrodes, Nat. Mater., 2012, 11, 306-310.

18 G. Feng and P. T. Cummings, Supercapacitor Capacitance Exhibits Oscillatory Behavior as a Function of Nanopore Size, J. Phys. Chem. Lett., 2011, 2, 2859-2864.

19 N. Jäckel, P. Simon, Y. Gogotsi and V. Presser, Increase in Capacitance by Subnanometer Pores in Carbon, ACS Energy Lett., 2016, 1, 1262-1265.

20 F. Stoeckli and T. A. Centeno, Centeno et al., Pore size distribution and capacitance in microporous carbons, Phys. Chem. Chem. Phys., 2012, 14, 11589-11591.

21 T. A. Centeno, O. Sereda and F. Stoeckli, Centeno et al., Capacitance in carbon pores of 0.7 to $15 \mathrm{~nm}$ : a regular pattern, Phys. Chem. Chem. Phys., 2011, 13, 12403-12406.

22 C. Merlet, B. Rotenberg, P. A. Madden, P.-L. Taberna, P. Simon, Y. Gogotsi and M. Salanne, On the molecular origin of supercapacitance in nanoporous carbon electrodes, Nat. Mater., 2012, 11, 306-310.

23 S. Kondrat, N. Georgi, M. V. Fedorov and A. A. Kornyshev, A superionic state in nano-porous double-layer capacitors: insights from Monte Carlo simulations, Phys. Chem. Chem. Phys., 2011, 13, 11359-11366.

24 C. Merlet, C. Péan, B. Rotenberg, P. A. Madden, B. Daffos, P. L. Taberna, P. Simon and M. Salanne, Highly confined ions store charge more efficiently in supercapacitors, Nat. Commun., 2013, 4, 2701.

25 C. Prehal, C. Koczwara, N. Jäckel, A. Schreiber, M. Burian, H. Amenitsch, M. A. Hartmann, V. Presser and O. Paris, Quantification of ion confinement and desolvation in nanoporous carbon supercapacitors with modelling and in situ X-ray scattering, Nat. Energy, 2017, 2, 16215.

26 J. Chmiola, C. Largeot, P.-L. Taberna, P. Simon and Y. Gogotsi, Desolvation of Ions in Subnanometer Pores and Its Effect on Capacitance and Double-Layer Theory, Angew. Chem., 2008, 120, 3440-3443.

27 Y. He, R. Qiao, J. Vatamanu, O. Borodin, D. Bedrov, J. Huang and B. G. Sumpter, Importance of Ion Packing on the Dynamics of Ionic Liquids during Micropore Charging, J. Phys. Chem. Lett., 2016, 7, 36-42.

28 L. Zhang, Y. Guo, K. Shen, J. Huo, Y. Liu and S. Guo, Ionmatching porous carbons with ultra-high surface area and superior energy storage performance for supercapacitors, J. Mater. Chem. A, 2019, 7, 9163-9172.

29 C. Koczwara, S. Rumswinkel, L. Hammerschmidt, M. Salihovic, M. S. Elsaesser, H. Amenitsch, O. Paris and N. Huesing, Nanofibers versus Nanopores: A Comparison of the Electrochemical Performance of Hierarchically Ordered Porous Carbons, ACS Appl. Energy Mater., 2019, 2, 5279-5291.

30 W. Schwieger, A. G. Machoke, T. Weissenberger, A. Inayat, T. Selvam, M. Klumpp and A. Inayat, Hierarchy concepts: classification and preparation strategies for zeolite containing materials with hierarchical porosity, Chem. Soc. Rev., 2016, 45, 3353-3376.

31 L. Borchardt, D. Leistenschneider, J. Haase and M. Dvoyashkin, Revising the Concept of Pore Hierarchy for Ionic Transport in Carbon Materials for Supercapacitors, Adv. Energy Mater., 2018, 8, 1800892.

32 P. Trogadas, V. Ramani, P. Strasser, T. F. Fuller and M.-O. Coppens, Hierarchically Structured Nanomaterials for Electrochemical Energy Conversion, Angew. Chem., Int. Ed., 2016, 55, 122-148.

33 Y. Liu, B. Huang, X. Lin and Z. Xie, Biomass-derived hierarchical porous carbons: boosting the energy density of supercapacitors via an ionothermal approach, J. Mater. Chem. A, 2017, 5, 13009-13018.

34 Y. An, Z. Li, Y. Yang, B. Guo, Z. Zhang, H. Wu and Z. Hu, Synthesis of Hierarchically Porous Nitrogen-Doped Carbon Nanosheets from Agaric for High-Performance Symmetric Supercapacitors, Adv. Mater. Interfaces, 2017, 4, 1700033.

35 S. Gadipelli, C. A. Howard, J. Guo, N. T. Skipper, H. Zhang, P. R. Shearing and D. J. L. Brett, Nanoporous Carbons: Superior Multifunctional Activity of Nanoporous Carbons with Widely Tunable Porosity: Enhanced Storage Capacities for Carbon-Dioxide, Hydrogen, Water, and Electric Charge, Adv. Energy Mater., 2020, 10, 1903649.

36 T. Liu, F. Zhang, Y. Song and Y. Li, Revitalizing carbon supercapacitor electrodes with hierarchical porous structures, J. Mater. Chem. A, 2017, 5, 17705-17733.

37 L. Zhou, K. Zhang, Z. Hu, Z. Tao, L. Mai, Y.-M. Kang, S.-L. Chou and J. Chen, Recent Developments on and Prospects for Electrode Materials with Hierarchical Structures for Lithium-Ion Batteries, Adv. Energy Mater., 2018, 8, 1701415.

38 Z. Yu, L. Tetard, L. Zhai and J. Thomas, Supercapacitor electrode materials: nanostructures from 0 to 3 dimensions, Energy Environ. Sci., 2015, 8, 702-730.

39 G. J. Lee and S. I. Pyun, Theoretical Approach to Ion Penetration into Pores with Pore Fractal Characteristics during Double-Layer Charging/Discharging on a Porous Carbon Electrode, Langmuir, 2006, 22, 10659-10665.

40 M. Salanne, B. Rotenberg, K. Naoi, K. Kaneko, P. L. Taberna, C. P. Grey, B. Dunn and P. Simon, Efficient storage mechanisms for building better supercapacitors, Nat. Energy, 2016, 1, 16070.

41 A. Belhboub, E. H. Lahrar, P. Simon and C. Merlet, On the development of an original mesoscopic model to predict 
the capacitive properties of carbon-carbon supercapacitors, Electrochim. Acta, 2019, 327, 135022.

42 F. W. Richey, B. Dyatkin, Y. Gogotsi and Y. A. Elabd, Ion Dynamics in Porous Carbon Electrodes in Supercapacitors Using in Situ Infrared Spectroelectrochemistry, J. Am. Chem. Soc., 2013, 135, 12818-12826.

43 J. M. Griffin, A. C. Forse, W. Y. Tsai, P. L. Taberna, P. Simon and C. P. Grey, In situ NMR and electrochemical quartz crystal microbalance techniques reveal the structure of the electrical double layer in supercapacitors, Nat. Mater., 2015, 14, 812.

44 Y. Wang, C. Malveau and D. Rochefort, Solid-state NMR and electrochemical dilatometry study of charge storage in supercapacitor with redox ionic liquid electrolyte, Energy Storage Mater., 2019, 20, 80-88.

45 M. D. Levi, N. Levy, S. Sigalov, G. Salitra, D. Aurbach and J. Maier, Electrochemical Quartz Crystal Microbalance (EQCM) Studies of Ions and Solvents Insertion into Highly Porous Activated Carbons, J. Am. Chem. Soc., 2010, 132, 13220-13222.

46 W. Y. Tsai, P. L. Taberna and P. Simon, Electrochemical Quartz Crystal Microbalance (EQCM) Study of Ion Dynamics in Nanoporous Carbons, J. Am. Chem. Soc., 2014, 136, 8722-8728.

47 D. T. L. Galhena, B. C. Bayer, S. Hofmann and G. A. J. Amaratunga, Understanding Capacitance Variation in Sub-nanometer Pores by in Situ Tuning of Interlayer Constrictions, ACS Nano, 2016, 10, 747-754.

48 F. W. Richey, C. Tran, V. Kalra and Y. A. Elabd, Ionic Liquid Dynamics in Nanoporous Carbon Nanofibers in Supercapacitors Measured with in Operando Infrared Spectroelectrochemistry, J. Phys. Chem. C, 2014, 118, 21846-21855.

49 M. V. Fedorov and A. A. Kornyshev, Ionic Liquids at Electrified Interfaces, Chem. Rev., 2014, 114, 2978-3036.

50 C. Merlet, B. Rotenberg, P. A. Madden, P.-L. Taberna, P. Simon, Y. Gogotsi and M. Salanne, On the molecular origin of supercapacitance in nanoporous carbon electrodes, Nat. Mater., 2012, 11, 306-310.

51 Y. Shim and H. J. Kim, Nanoporous Carbon Supercapacitors in an Ionic Liquid: A Computer Simulation Study, ACS Nano, 2010, 4, 2345-2355.

52 L. Hao, X. Li and L. Zhi, Carbonaceous Electrode Materials for Supercapacitors, Adv. Mater., 2013, 25, 3899-3904.

53 D. Y. Shin, K. W. Sung and H. J. Ahn, Synergistic effect of heteroatom-doped activated carbon for ultrafast charge storage kinetics, Appl. Surf. Sci., 2019, 478, 499-504.

54 H. Peng, B. Yao, X. Wei, T. Liu, T. Kou, P. Xiao, Y. Zhang and Y. Li, Pore and Heteroatom Engineered Carbon Foams for Supercapacitors, Adv. Energy Mater., 2019, 9, 1803665.

55 G. Zou, H. Hou, C. W. Foster, C. E. Banks, T. Guo, Y. Jiang, Y. Zhang and X. Ji, Advanced Hierarchical Vesicular Carbon Co-Doped with S, P, N for High-Rate Sodium Storage, Adv. Sci., 2018, 5, 1800241.

56 L. Zhao, L. Z. Fan, M. Q. Zhou, H. Guan, S. Qiao, M. Antonietti and M.-M. Titirici, Nitrogen-Containing Hydrothermal Carbons with Superior Performance in Supercapacitors, Adv. Mater., 2010, 22, 5202-5206.
57 P. Yan, B. Zhang, K.-H. Wu, D. Su and W. Qi, Surface chemistry of nanocarbon: Characterization strategies from the viewpoint of catalysis and energy conversion, Carbon, 2019, 143, 915-936.

58 M. R. Benzigar, S. N. Talapaneni, S. Joseph, K. Ramadass, G. Singh, J. Scaranto, U. Ravon, K. Al-Bahily and A. Vinu, Recent advances in functionalized micro and mesoporous carbon materials: synthesis and applications, Chem. Soc. Rev., 2018, 47, 2680-2721.

59 S. Roldán, C. Blanco, M. Granda, R. Menéndez and R. Santamaría, Towards a Further Generation of HighEnergy Carbon-Based Capacitors by Using Redox-Active Electrolytes, Angew. Chem., Int. Ed., 2011, 50, 1699-1701.

60 P. Azaïs, L. Duclaux, P. Florian, D. Massiot, M.-A. LilloRodenas, A. Linares-Solano, J.-P. Peres, C. Jehoulet and F. Béguin, Causes of supercapacitors ageing in organic electrolyte, J. Power Sources, 2007, 171, 1046-1053.

61 F. Wei, H. F. Zhang, H. E. Xiao-jun, M. A. Hao, S. A. Dong and X. Y. Xie, Synthesis of porous carbons from coal tar pitch for high-performance supercapacitors, New Carbon Mater., 2019, 34(2), 132-139.

62 M. Inagaki, M. Toyoda, Y. Soneda and T. Morishita, Nitrogendoped carbon materials, Carbon, 2018, 132, 104-140.

63 Z. Zhang, L. Wang, Y. Li, Y. Wang, J. Zhang, G. Guan, Z. Pan, G. Zheng and H. Peng, Nitrogen-Doped CoreSheath Carbon Nanotube Array for Highly Stretchable Supercapacitor, Adv. Energy Mater., 2017, 7, 1601814.

64 Z. Bi, L. Huo, Q. Kong, F. Li, J. Chen, A. Ahmad, X. Wei, L. Xie and C.-M. Chen, Structural Evolution of Phosphorus Species on Graphene with a Stabilized Electrochemical Interface, ACS Appl. Mater. Interfaces, 2019, 11, 11421-11430.

65 J. Patino, N. Lopez-Salas, M. C. Gutierrez, D. Carriazo, M. L. Ferrer and F.d. Monte, Phosphorus-doped carboncarbon nanotube hierarchical monoliths as true threedimensional electrodes in supercapacitor cells, J. Mater. Chem. A, 2016, 4, 1251-1263.

66 H. Chen, Y. Xiong, T. Yu, P. Zhu, X. Yan, Z. Wang and S. Guan, Boron and nitrogen co-doped porous carbon with a high concentration of boron and its superior capacitive behavior, Carbon, 2017, 113, 266-273.

67 K. Liu, J. Xu, Y. Wang, M. Qian, W. Zhao, Y. Zeng and F. Huang, Boron and Nitrogen Co-Doped Trimodal-Porous Wood-Derived Carbon for Boosting Capacitive Performance, Energy Technol., 2019, 8, 1900950.

68 A. M. Puziy, O. I. Poddubnaya, B. Gawdzik and J. M. D. Tascón, Phosphorus-containing carbons: preparation, properties and utilization, Carbon, 2020, 157, 796-846.

69 D. W. Wang, F. Li, Z. G. Chen, G. Q. Lu and H. M. Cheng, Synthesis and Electrochemical Property of Boron-Doped Mesoporous Carbon in Supercapacitor, Chem. Mater., 2008, 20, 7195-7200.

70 G. Ren, Y. Li, Q. Chen, Y. Qian, J. Zheng, Y. Zhu and C. Teng, Sepia-Derived N, P Co-doped Porous Carbon Spheres as Oxygen Reduction Reaction Electrocatalyst and Supercapacitor, ACS Sustainable Chem. Eng., 2018, 6, 16032-16038. 
71 H. Jin, X. Feng, J. Li, M. Li, Y. Xia, Y. Yuan, C. Yang, B. Dai, Z. Lin, J. Wang, J. Lu and S. Wang, Heteroatom-Doped Porous Carbon Materials with Unprecedented High Volumetric Capacitive Performance, Angew. Chem., Int. Ed., 2019, 58, 2397-2401.

72 M. Liu, J. Niu, Z. Zhang, M. Dou and F. Wang, Potassium compound-assistant synthesis of multi-heteroatom doped ultrathin porous carbon nanosheets for high performance supercapacitors, Nano Energy, 2018, 51, 366-372.

73 H. Wang, A. C. Forse, J. M. Griffin, N. M. Trease, L. Trognko, P.-L. Taberna, P. Simon and C. P. Grey, In Situ NMR Spectroscopy of Supercapacitors: Insight into the Charge Storage Mechanism, J. Am. Chem. Soc., 2013, 135, 18968-18980.

74 L. Hao, X. Li and L. Zhi, Carbonaceous Electrode Materials for Supercapacitors, Adv. Mater., 2013, 25, 3899-3904.

75 H. Helmholtz, Ueber einige Gesetze der Vertheilung elektrischer Ströme in körperlichen Leitern mit Anwendung auf die thierisch-elektrischen Versuche, Ann. Phys., 1853, 165, 211-233.

76 H. Helmholtz, Studien über electrische Grenzschichten, Ann. Phys., 1879, 243, 337-382.

77 A. Noori, M. F. El-Kady, M. S. Rahmanifar, R. B. Kaner and M. F. Mousavi, Towards establishing standard performance metrics for batteries, supercapacitors and beyond, Chem. Soc. Rev., 2019, 48, 1272-1341.

$78 \mathrm{H}$. Shi, Activated carbons and double layer capacitance, Electrochim. Acta, 1996, 41, 1633-1639.

79 T. E. Rufford, D. Hulicova-Jurcakova, Z. Zhu and G. Q. Lu, Empirical Analysis of the Contributions of Mesopores and Micropores to the Double-Layer Capacitance of Carbons, J. Phys. Chem. C, 2009, 113, 19335-19343.

80 J. Huang, B. G. Sumpter and V. Meunier, Theoretical Model for Nanoporous Carbon Supercapacitors, Angew. Chem., Int. Ed., 2008, 47, 520-524.

81 L. Yang, B. H. Fishbine, A. Migliori and L. R. Pratt, Molecular Simulation of Electric Double-Layer Capacitors Based on Carbon Nanotube Forests, J. Am. Chem. Soc., 2009, 131, 12373-12376.

82 K. Ma, C. Zhang, C. E. Woodward and X. Wang, Bridging the gap between macroscopic electrochemical measurements and microscopic molecular dynamic simulations: Porous carbon supercapacitor with ionic liquids, Electrochim. Acta, 2018, 289, 29-38.

83 J. M. Griffin, A. C. Forse, H. Wang, N. M. Trease, P. L. Taberna, P. Simon and C. P. Grey, Ion counting in supercapacitor electrodes using NMR spectroscopy, Faraday Discuss., 2014, 176, 49-68.

84 J. Chmiola, C. Largeot, P.-L. Taberna, P. Simon and Y. Gogotsi, Desolvation of Ions in Subnanometer Pores and Its Effect on Capacitance and Double-Layer Theory, Angew. Chem., Int. Ed., 2008, 47, 3392-3395.

85 M. D. Levi, S. Sigalov, D. Aurbach and L. Daikhin, In Situ Electrochemical Quartz Crystal Admittance Methodology for Tracking Compositional and Mechanical Changes in Porous Carbon Electrodes, J. Phys. Chem. C, 2013, 117, 14876-14889.
86 N. F. Berk, Scattering properties of a model bicontinuous structure with a well defined length scale, Phys. Rev. Lett., 1987, 58, 2718-2721.

87 C. Prehal, C. Koczwara, N. Jäckel, H. Amenitsch, V. Presser and O. Paris, A carbon nanopore model to quantify structure and kinetics of ion electrosorption with in situ smallangle X-ray scattering, Phys. Chem. Chem. Phys., 2017, 19, 15549-15561.

88 G. Oukali, E. Salager, M. R. Ammar, C.-E. Dutoit, V. SarouKanian, P. Simon, E. Raymundo-Piñero and M. Deschamps, In Situ Magnetic Resonance Imaging of a Complete Supercapacitor Giving Additional Insight on the Role of Nanopores, ACS Nano, 2019, 13, 12810-12815.

89 X. Wang, A. Y. Mehandzhiyski, B. Arstad, K. L. Van Aken, T. S. Mathis, A. Gallegos, Z. Tian, D. Ren, E. Sheridan, B. A. Grimes, D. Jiang, J. Wu, Y. Gogotsi and D. Chen, Selective Charging Behavior in an Ionic Mixture Electrolyte-Supercapacitor System for Higher Energy and Power, J. Am. Chem. Soc., 2017, 139, 18681-18687.

90 J. P. Paraknowitsch and A. Thomas, Doping carbons beyond nitrogen: an overview of advanced heteroatom doped carbons with boron, sulphur and phosphorus for energy applications, Energy Environ. Sci., 2013, 6, 2839-2855.

91 Z. Yang, Z. Yao, G. Li, G. Fang, H. Nie, Z. Liu, X. Zhou, X.a. Chen and S. Huang, Sulfur-Doped Graphene as an Efficient Metal-free Cathode Catalyst for Oxygen Reduction, ACS Nano, 2012, 6, 205-211.

92 H. Wang, R. Fan, J. Miao, J. Deng and Y. Wang, Oxygen Groups Immobilized on Micropores for Enhancing the Pseudocapacitance, ACS Sustainable Chem. Eng., 2019, 7, 11407-11414.

93 X. Fan, Y. Lu, H. Xu, X. Kong and J. Wang, Reversible redox reaction on the oxygen-containing functional groups of an electrochemically modified graphite electrode for the pseudo-capacitance, J. Mater. Chem., 2011, 21, 18753-18760.

94 G. Lota, T. A. Centeno, E. Frackowiak and F. Stoeckli, Improvement of the structural and chemical properties of a commercial activated carbon for its application in electrochemical capacitors, Electrochim. Acta, 2008, 53, 2210-2216.

95 Y. He, Y. Zhang, X. Li, Z. Lv, X. Wang, Z. Liu and X. Huang, Capacitive mechanism of oxygen functional groups on carbon surface in supercapacitors, Electrochim. Acta, 2018, 282, 618-625.

96 S. Kundu, Y. Wang, W. Xia and M. Muhler, Thermal Stability and Reducibility of Oxygen-Containing Functional Groups on Multiwalled Carbon Nanotube Surfaces: A Quantitative High-Resolution XPS and TPD/TPR Study, J. Phys. Chem. C, 2008, 112, 16869-16878.

97 D. Rosenthal, M. Ruta, R. Schlögl and L. Kiwi-Minsker, Combined XPS and TPD study of oxygen-functionalized carbon nanofibers grown on sintered metal fibers, Carbon, 2010, 48, 1835-1843.

98 H. Oda, A. Yamashita, S. Minoura, M. Okamoto and T. Morimoto, Modification of the oxygen-containing 
functional group on activated carbon fiber in electrodes of an electric double-layer capacitor, J. Power Sources, 2006, 158, 1510-1516.

99 D. Zhang, J. Wang, C. He, Y. Wang, T. Guan, J. Zhao, J. Qiao and K. Li, Rational Surface Tailoring Oxygen Functional Groups on Carbon Spheres for Capacitive Mechanistic Study, ACS Appl. Mater. Interfaces, 2019, 11, 13214-13224.

100 M. D. Zhang, C. Yu, Z. Ling, J. H. Yu, S. F. Li, C. T. Zhao, H. L. Huang and J. S. Qiu, A recyclable route to produce biochar with a tailored structure and surface chemistry for enhanced charge storage, Green Chem., 2019, 21, 2095-2103.

101 S. T. Yuan, X. H. Huang, H. Wang, L. J. Xie, J. Y. Cheng, Q. Q. Kong, G. H. Sun and C. M. Chen, J. Energy Chem., 2020, 51, 396-404.

102 S. M. Li, S. Y. Yang, Y. S. Wang, H. P. Tsai, H. W. Tien, S. T. Hsiao, W. H. Liao, C. L. Chang, C. C. M. Ma and C. C. $\mathrm{Hu}, \mathrm{N}$-doped structures and surface functional groups of reduced graphene oxide and their effect on the electrochemical performance of supercapacitor with organic electrolyte, J. Power Sources, 2015, 278, 218-229.

103 J. Y. Cheng, Z. L. Yi, Z. B. Wang, F. Li, N. N. Gong, A. Ahmad, X. Q. Guo, G. Song, S. T. Yuan and C. M. Chen, Towards optimized Li-ion storage performance: Insight on the oxygen species evolution of hard carbon by $\mathrm{H} 2$ reduction, Electrochim. Acta, 2020, 337, 135736.

104 G. Moussa, C. Matei Ghimbeu, P.-L. Taberna, P. Simon and C. Vix-Guterl, Relationship between the carbon nanoonions (CNOs) surface chemistry/defects and their capacitance in aqueous and organic electrolytes, Carbon, 2016, 105, 628-637.

105 M. Cheng, Y. Meng, Q. Meng, L. Mao, M. Zhang, K. Amin, A. Ahmad, S. Wu and Z. Wei, A hierarchical porous $\mathrm{N}$-doped carbon electrode with superior rate performance and cycling stability for flexible supercapacitors, Mater. Chem. Front., 2018, 2, 986-992.

106 K. Gong, F. Du, Z. Xia, M. Durstock and L. Dai, NitrogenDoped Carbon Nanotube Arrays with High Electrocatalytic Activity for Oxygen Reduction, Science, 2009, 323, 760-764.

107 K. Jurewicz, K. Babeł, A. Źiółkowski and H. Wachowska, Ammoxidation of active carbons for improvement of supercapacitor characteristics, Electrochim. Acta, 2003, 48, 1491-1498.

108 K. Liu, X. Zheng, K. Wang, C. Wang and M. Chen, Sodium metal-assisted carbonization of pyrrole to prepare N-doped porous carbons for high-rate performance supercapacitors, Carbon, 2019, 153, 265-273.

109 F. Sun, H. Wu, X. Liu, F. Liu, H. Zhou, J. Gao and Y. Lu, Nitrogen-rich carbon spheres made by a continuous spraying process for high-performance supercapacitors, Nano Res., 2016, 9, 3209-3221.

110 D. W. Wang, F. Li, L. C. Yin, X. Lu, Z. G. Chen, I. R. Gentle, G. Q. Lu and H. M. Cheng, Nitrogen-Doped Carbon Monolith for Alkaline Supercapacitors and Understanding Nitrogen-Induced Redox Transitions, Chem. - Eur. J., 2012, 18(17), 5345-5351.
111 L. Zhao, L. Z. Fan, M. Q. Zhou, H. Guan, S. Qiao, M. Antonietti and M.-M. Titirici, Nitrogen-Containing Hydrothermal Carbons with Superior Performance in Supercapacitors, Adv. Mater., 2010, 22, 5202-5206.

112 A. Olejniczak, M. Leżańska, A. Pacuła, P. Nowak, J. Włoch and J. P. Łukaszewicz, Nitrogen-containing mesoporous carbons with high capacitive properties derived from a gelatin biomolecule, Carbon, 2015, 91, 200-214.

113 Z. Li, L. Zhang, B. S. Amirkhiz, X. Tan, Z. Xu, H. Wang, B. C. Olsen, C. M. B. Holt and D. Mitlin, Carbonized Chicken Eggshell Membranes with 3D Architectures as High-Performance Electrode Materials for Supercapacitors, Adv. Energy Mater., 2012, 2, 431-437.

114 D. Salinas-Torres, S. Shiraishi, E. Morallón and D. CazorlaAmorós, Improvement of carbon materials performance by nitrogen functional groups in electrochemical capacitors in organic electrolyte at severe conditions, Carbon, 2015, 82, 205-213.

115 O. Ornelas, J. M. Sieben, R. Ruiz-Rosas, E. Morallón, D. Cazorla-Amorós, J. Geng, N. Soin, E. Siores and B. F. G. Johnson, On the origin of the high capacitance of nitrogencontaining carbon nanotubes in acidic and alkaline electrolytes, Chem. Commun., 2014, 50, 11343-11346.

116 D. Hulicova, M. Kodama and H. Hatori, Electrochemical performance of nitrogen-enriched carbons in aqueous and non-aqueous supercapacitors, Chem. Mater., 2006, 18, 2318-2326.

117 D. Salinas-Torres, S. Shiraishi, E. Morallón and D. CazorlaAmorós, Improvement of carbon materials performance by nitrogen functional groups in electrochemical capacitors in organic electrolyte at severe conditions, Carbon, 2015, 82, 205-213.

118 S. M. Li, S. Y. Yang, Y. S. Wang, H. P. Tsai, H. W. Tien, S. T. Hsiao, W. H. Liao, C. L. Chang, C. C. M. Ma and C. C. $\mathrm{Hu}, \mathrm{N}$-doped structures and surfac e functional group $\mathrm{s}$ of red uced graphe ne oxide and their effec $t$ on the electrochemical pe rformance of supercapacitor with organic electrolyte, J. Power Sources, 2015, 278, 218-229.

119 D. S. Yang, D. Bhattacharjya, S. Inamdar, J. Park and J. S. Yu, Phosphorus-Doped Ordered Mesoporous Carbons with Different Lengths as Efficient Metal-Free Electrocatalysts for Oxygen Reduction Reaction in Alkaline Media, J. Am. Chem. Soc., 2012, 134, 16127-16130.

120 C. Huang, T. Sun and D. Hulicova-Jurcakova, Wide Electrochemical Window of Supercapacitors from Coffee Bean-Derived Phosphorus-Rich Carbons, ChemSusChem, 2013, 6, 2330-2339.

121 V. Schwartz, H. Xie, H. M. Meyer III, S. H. Overbury and C. Liang, Oxidative dehydrogenation of isobutane on phosphorous-modified graphitic mesoporous carbon, Carbon, 2011, 49, 659-668.

122 R. Imamura, K. Matsui, S. Takeda, J. Ozaki and A. Oya, A new role for phosphorus in graphitization of phenolic resin, Carbon, 1999, 37, 261-267.

123 S. Bourbigot, M. Le Bras, R. Delobel, P. Bréant and J.m. TréMillon, Carbonization mechanisms resulting from 
intumescence-part II. Association with an ethylene terpolymer and the ammonium polyphosphate-pentaerythritol fire retardant system, Carbon, 1995, 33, 283-294.

124 M. Jagtoyen and F. Derbyshire, Activated carbons from yellow poplar and white oak by $\mathrm{H}_{3} \mathrm{PO}_{4}$ activation, Carbon, 1998, 36, 1085-1097.

125 D. Hulicova-Jurcakova, A. M. Puziy, O. I. Poddubnaya, F. Suarez-Garcia, J. M. D. Tascon and G. Q. Lu, Highly Stable Performance of Supercapacitors from PhosphorusEnriched Carbons, J. Am. Chem. Soc., 2009, 131, 5026-5027.

126 J. Yi, Y. Qing, C. Wu, Y. Zeng, Y. Wu, X. Lu and Y. Tong, Lignocellulose-derived porous phosphorus-doped carbon as advanced electrode for supercapacitors, J. Power Sources, 2017, 351, 130-137.

127 X. Yu, H. J. Kim, J. Y. Hong, Y. M. Jung, K. D. Kwon, J. Kong and H. S. Park, Elucidating surface redox charge storage of phosphorus-incorporated graphenes with hierarchical architectures, Nano Energy, 2015, 15, 576-586.

128 W. Ma, L. Xie, L. Dai, G. Sun, J. Chen, F. Su, Y. Cao, H. Lei, Q. Kong and C. M. Chen, Influence of phosphorus doping on surface chemistry and capacitive behaviors of porous carbon electrode, Electrochim. Acta, 2018, 266, 420-430.

129 S. Shiraishi, M. Kibe, T. Yokoyama, H. Kurihara, N. Patel, A. Oya, Y. Kaburagi and Y. Hishiyama, Electric double layer capacitance of multi-walled carbon nanotubes and B-doping effect, Appl. Phys. A: Mater. Sci. Process., 2006, 82, 585-591.

130 L. Niu, Z. Li, W. Hong, J. Sun, Z. Wang, L. Ma, J. Wang and S. Yang, Pyrolytic synthesis of boron-doped graphene and its application as electrode material for supercapacitors, Electrochim. Acta, 2013, 108, 666-673.

131 M. Enterria, M. F. R. Pereira, J. I. Martins and J. L. Figueiredo, Hydrothermal functionalization of ordered mesoporous carbons: The effect of boron on supercapacitor performance, Carbon, 2015, 95, 72-83.

132 P. Ayala, J. Reppert, M. Grobosch, M. Knupfer, T. Pichler and A. M. Rao, Evidence for substitutional boron in doped single-walled carbon nanotubes, Appl. Phys. Lett., 2010, 96, 183110.

133 L. S. Panchokarla, K. S. Subrahmanyam, S. K. Saha, A. Govindaraj, H. R. Krishnamurthy, U. V. Waghmare and C. N. R. Rao, Synthesis, Structure and Properties of Boron- and Nitrogen-Doped Graphene, Adv. Mater., 2009, 21, 4726.

134 T. Lin, F. Huang, J. Liang and Y. Wang, A facile preparation route for boron-doped graphene, and its CdTe solar cell application, Energy Environ. Sci., 2011, 4, 862-865.

135 J. Gao, X. Wang, Y. Zhang, J. Liu, Q. Lu and M. Liu, Borondoped ordered mesoporous carbons for the application of supercapacitors, Electrochim. Acta, 2016, 207, 266-274.

136 X. Li, L. Fan, Z. Li, K. Wang, M. Zhong, J. Wei, D. Wu and H. Zhu, Boron Doping of Graphene for Graphene-Silicon p-n Junction Solar Cells, Adv. Energy Mater., 2012, 2, 425-429.

137 Y. B. Tang, L. C. Yin, Y. Yang, X. H. Bo, Y. L. Cao, H. E. Wang, W.-J. Zhang, I. Bello, S. T. Lee, H. M. Cheng and C. S. Lee, Tunable Band Gaps and p-Type Transport Properties of Boron-Doped Graphenes by Controllable Ion Doping Using Reactive Microwave Plasma, ACS Nano, 2012, 6, 1970-1978.

138 D. Y. Yeom, W. Jeon, N. D. K. Tu, S. Y. Yeo, S.-S. Lee, B. J. Sung, H. Chang, J. A. Lim and H. Kim, Highconcentration boron doping of graphene nanoplatelets by simple thermal annealing and their supercapacitive properties, Sci. Rep., 2015, 5, 9817.

139 R. Nankya, J. Lee, D. O. Opar and H. Jung, Electrochemical behavior of boron-doped mesoporous graphene depending on its boron configuration, Appl. Surf. Sci., 2019, 489, $552-559$.

140 S. S. Balaji, M. Karnan, J. Kamarsamam and M. Sathish, Synthesis of Boron-Doped Graphene by Supercritical Fluid Processing and its Application in Symmetric Supercapacitors using Various Electrolytes, ChemElectroChem, 2019, 6(5), 1492-1499.

141 H. Zhu, X. Wang, X. Liu and X. Yang, Integrated Synthesis of Poly(o-phenylenediamine)-Derived Carbon Materials for High Performance Supercapacitors, Adv. Mater., 2012, 24, 6524-6529.

142 L.-Z. Fan, S. Qiao, W. Song, M. Wu, X. He and X. Qu, Effects of the functional groups on the electrochemical properties of ordered porous carbon for supercapacitors, Electrochim. Acta, 2013, 105, 299-304.

143 J. Wang, F. Qin, Z. Guo and W. Shen, Oxygen- and Nitrogen-Enriched Honeycomb-Like Porous Carbon from Laminaria japonica with Excellent Supercapacitor Performance in Aqueous Solution, ACS Sustainable Chem. Eng., 2019, 7, 11550-11563.

144 R. Zhang, X. Jing, Y. Chu, L. Wang, W. Kang, D. Wei, H. Li and S. Xiong, Nitrogen/oxygen co-doped monolithic carbon electrodes derived from melamine foam for highperformance supercapacitors, J. Mater. Chem. A, 2018, 6, 17730-17739.

145 K. Liang, C. Ma, L. Liu, Y. Yu, H. Lv, Y. Zhang and A. Chen, Nitrogen and oxygen co-doped ordered dual-mesoporous carbon for supercapacitors, J. Alloys Compd., 2019, 805, 859-867.

146 D. Hulicova-Jurcakova, M. Seredych, G. Q. Lu and T. J. Bandosz, Combined Effect of Nitrogen- and OxygenContaining Functional Groups of Microporous Activated Carbon on its Electrochemical Performance in Supercapacitors, Adv. Funct. Mater., 2009, 19, 438-447.

147 Z. Song, L. Miao, L. Li, D. Zhu, Y. Lv, W. Xiong, H. Duan, Z. Wang, L. Gan and M. Liu, A universal strategy to obtain highly redox-active porous carbons for efficient energy storage, J. Mater. Chem. A, 2020, 8, 3717-3725.

148 Y. Wang, B. Ding, D. Guo and X. Wu, A novel way to synthesize nitrogen and oxygen co-doped porous carbon for high performance supercapacitors, Microporous Mesoporous Mater., 2019, 282, 114-120.

149 H. Zhao, B. Xing, C. Zhang, G. Huang, Q. Liu, G. Yi, J. Jia, M. Ma, Z. Chen and C. Zhang, Efficient synthesis of nitrogen and oxygen co-doped hierarchical porous carbons 
derived from soybean meal for high-performance supercapacitors, J. Alloys Compd., 2018, 766, 705-715.

150 C. H. Choi, S. H. Park and S. I. Woo, Binary and Ternary Doping of Nitrogen,Boron, and $\mathrm{P}$ hosphorus i nto $\mathrm{C}$ arbon for Enhancing Electrochemical Oxygen Reduction Activity, ACS Nano, 2012, 6, 7084-7091.

151 X. Yan, Y. Yu, S.-K. Ryu, J. Lan, X. Jia and X. Yang, Simple and scalable synthesis of phosphorus and nitrogen enriched porous carbons with high volumetric capacitance, Electrochim. Acta, 2014, 136, 466-472.

152 J. Jin, X. Qiao, F. Zhou, Z.-S. Wu, L. Cui and H. Fan, Interconnected Phosphorus and Nitrogen Codoped Porous Exfoliated Carbon Nanosheets for High-Rate Supercapacitors, ACS Appl. Mater. Interfaces, 2017, 9(20), 17317-17325.

153 Y. Ma, D. Wu, T. Wang and D. Jia, Phosphorus Co-doped Carbon Obtained from Amino Acid Based Resin Xerogel as Efficient Electrode for Supercapacitor, ACS Appl. Energy Mater., 2020, 3, 957-969.

154 Y. P. Zhu, Y. Jing, A. Vasileff, T. Heine and S. Z. Qiao, 3D Synergistically Active Carbon Nanofibers for Improved Oxygen Evolution, Adv. Energy Mater., 2017, 7, 1602928.

155 X. Zhao, S. Wang and Q. Wu, Nitrogen and phosphorus dual-doped hierarchical porous carbon with excellent supercapacitance performance, Electrochim. Acta, 2017, 247, 1140-1146.

156 J. Chen, H. Wei, H. Chen, W. Yao, H. Lin and S. Han, N/P co-doped hierarchical porous carbon materials for superior performance supercapacitors, Electrochim. Acta, 2018, 271, 49-57.

157 S. Huo, Y. Zhao, M. Zong, B. Liang, X. Zhang, I. U. Khan, $\mathrm{X}$. Song and K. Li, Boosting supercapacitor and capacitive deionization performance of hierarchically porous carbon by polar surface and structural engineering, J. Mater. Chem. A, 2020, 8, 2505-2517.

158 M. Li, Z. Bi, L. Xie, G. Sun, Z. Liu, Q. Kong, X. Wei and C.-M. Chen, From Starch to Carbon Materials: Insight into the Cross-Linking Reaction and Its Influence on the Carbonization Process, ACS Sustainable Chem. Eng., 2019, 7, 14796-14804.

159 Y. Lu, Z. Li, Z. Bai, H. Mi, C. Ji, H. Pang, C. Yu and J. Qiu, High energy-power Zn-ion hybrid supercapacitors enabled by layered B/N co-doped carbon cathode, Nano Energy, 2019, 66, 104132.

160 L. Zhang, G. Xia, Z. Guo, D. Sun, X. Li and X. Yu, In situ fabrication of three-dimensional nitrogen and boron co-doped porous carbon nanofibers for high performance lithium-ion batteries, J. Power Sources, 2016, 324, 294-301.

161 X. Zou, D. Wu, Y. Mu, L. Xing, W. Zhang, Z. Gao, F. Xu and K. Jiang, Boron and nitrogen Co-doped holey graphene aerogels with rich B-N motifs for flexible supercapacitors, Carbon, 2020, 159, 94-101.

162 E. Iyyamperumal, S. Wang and L. Dai, Vertically Aligned BCN Nanotubes with High Capacitance, ACS Nano, 2012, 6, 5259-5265.
163 S. Qiu, Z. Chen, H. Zhuo, Y. Hu, Q. Liu, X. Peng and L. Zhong, Using $\mathrm{FeCl}_{3}$ as a Solvent, Template, and Activator To Prepare B, N Co-Doping Porous Carbon with Excellent Supercapacitance, ACS Sustainable Chem. Eng., 2019, 7, 15983-15994.

164 J. Hao, J. Wang, S. Qin, D. Liu, Y. Li and W. Lei, B/N co-doped carbon nanosphere frameworks as highperformance electrodes for supercapacitors, J. Mater. Chem. A, 2018, 6, 8053-8058.

165 D. Guo, B. Ding, X. Hu, Y. Wang, F. Han and X. Wu, Synthesis of Boron and Nitrogen Codoped Porous Carbon Foam for High Performance Supercapacitors, ACS Sustainable Chem. Eng., 2018, 6, 11441-11449.

166 Z. Zhao and Y. Xie, Electrochemical supercapacitor performance of boron and nitrogen co-doped porous carbon nanowires, J. Power Sources, 2018, 400, 264-276.

167 W. Yang, W. Yang, A. Song, L. Gao, L. Su and G. Shao, Supercapacitance of nitrogen-sulfur-oxygen co-doped 3D hierarchical porous carbon in aqueous and organic electrolyte, J. Power Sources, 2017, 359, 556-567.

168 L. Wang, Z. Sofer and M. Pumera, Will Any Crap We Put into Graphene Increase Its Electrocatalytic Effect?, ACS Nano, 2020, 14, 21-25.

169 M. Liu, J. Niu, Z. Zhang, M. Dou and F. Wang, Potassium compound-assistant synthesis of multi-heteroatom doped ultrathin porous carbon nanosheets for high performance supercapacitors, Nano Energy, 2018, 51, 366-372.

170 Y. Ma, X. Zhang, Z. Liang, C. Wang, Y. Sui, B. Zheng, Y. Ye, W. Ma, Q. Zhao and C. Qin, B/P/N/O co-doped hierarchical porous carbon nanofiber self-standing film with high volumetric and gravimetric capacitance performances for aqueous supercapacitors, Electrochim. Acta, 2020, 337, 135800.

171 H. Peng, B. Yao, X. Wei, T. Liu, T. Kou, P. Xiao, Y. Zhang and Y. Li, Pore and Heteroatom Engineered Carbon Foams for Supercapacitors, Adv. Energy Mater., 2019, 9, 1803665.

172 H. Jin, X. Feng, J. Li, M. Li, Y. Xia, Y. Yuan, C. Yang, B. Dai, Z. Lin, J. Wang, J. Lu and S. Wang, Heteroatom-Doped Porous Carbon Materials with Unprecedented High Volumetric Capacitive Performance, Angew. Chem., Int. Ed., 2019, 58, 2397-2401.

173 Y. Zhao, S. Huang, M. Xia, S. Rehman, S. Mu, Z. Kou, Z. Zhang, Z. Chen, F. Gao and Y. Hou, N-P-O co-doped high performance $3 \mathrm{D}$ graphene prepared through red phosphorous-assisted "cutting-thin" technique: A universal synthesis and multifunctional applications, Nano Energy, 2016, 28, 346-355.

174 F. Liu, Z. Wang, H. Zhang, L. Jin, X. Chu, B. Gu, H. Huang and W. Yang, Nitrogen, oxygen and sulfur co-doped hierarchical porous carbons toward high-performance supercapacitors by direct pyrolysis of kraft lignin, Carbon, 2019, 149, 105-116.

175 W. Zhou, S. Lei, S. Sun, X. Ou, Q. Fu, Y. Xu, Y. Xiao and B. Cheng, From weed to multi-heteroatom-doped honeycomblike porous carbon for advanced supercapacitors: A gelatinization-controlled one-step carbonization, J. Power Sources, 2018, 402, 203-212. 
176 L. Wan, X. Li, N. Li, M. Xie, C. Du, Y. Zhang and J. Chen, Multi-heteroatom-doped hierarchical porous carbon derived from chestnut shell with superior performance in supercapacitors, J. Alloys Compd., 2019, 790, 760-771.

177 G. Zhao, Y. Li, G. Zhu, J. Shi, T. Lu and L. Pan, BiomassBased N, P, and S Self-Doped Porous Carbon for
High-Performance Supercapacitors, ACS Sustainable Chem. Eng., 2019, 7, 12052-12060.

178 H. Li, L. L. Ren, D. Ai, Z. B. Han, Y. Liu, B. Yao and Q. Wang, Ternary polymer nanocomposites with concurrently enhanced dielectric constant and breakdown strength for hightemperature electrostatic capacitors, InfoMat, 2020, 2, 389-400. 